\title{
Article \\ Accurate Photovoltaic Models Based on an Adaptive Opposition Artificial Hummingbird Algorithm
}

\author{
Abdelhady Ramadan ${ }^{1}$, Salah Kamel ${ }^{1} \mathbb{D}$, Mohamed H. Hassan ${ }^{1} \mathbb{D}$, Emad M. Ahmed ${ }^{2, *}$ (D) and Hany M. Hasanien ${ }^{3} \mathbb{D}$ \\ 1 Department of Electrical Engineering, Faculty of Engineering, Aswan University, Aswan 81542, Egypt; \\ eng.abdalhady@gmail.com (A.R.); skamel@aswu.edu.eg (S.K.); mohamedhosnymoee@gmail.com (M.H.H.) \\ 2 Department of Electrical Engineering, College of Engineering, Jouf University, Sakaka 72388, Saudi Arabia \\ 3 Electrical Power and Machines Department, Faculty of Engineering, Ain Shams University, \\ Cairo 11517, Egypt; hanyhasanien@ieee.org \\ * Correspondence: emamahmoud@ju.edu.sa
}

check for

updates

Citation: Ramadan, A.; Kamel, S.;

Hassan, M.H.; Ahmed, E.M.;

Hasanien, H.M. Accurate

Photovoltaic Models Based on an

Adaptive Opposition Artificial

Hummingbird Algorithm. Electronics

2022, 11, 318. https://doi.org/

10.3390/electronics 11030318

Academic Editor:

Enrique Rosales-Asensio

Received: 17 December 2021

Accepted: 17 January 2022

Published: 20 January 2022

Publisher's Note: MDPI stays neutral with regard to jurisdictional claims in published maps and institutional affiliations.

Copyright: (c) 2022 by the authors. Licensee MDPI, Basel, Switzerland. This article is an open access article distributed under the terms and conditions of the Creative Commons Attribution (CC BY) license (https:/ / creativecommons.org/licenses/by/ $4.0 /)$.

\begin{abstract}
The greater the demand for energy, the more important it is to improve and develop permanent energy sources, because of their advantages over non-renewable energy sources. With the development of artificial intelligence algorithms and the presence of so many data, the evolution of simulation models has increased. In this research, an improvement to one recent optimization algorithm called the artificial hummingbird algorithm (AHA) is proposed. An adaptive opposition approach is suggested to select whether or not to use an opposition-based learning (OBL) method. This improvement is developed based on adding an adaptive updating mechanism to enable the original algorithm to obtain more accurate results with more complex problems, and is called the adaptive opposition artificial hummingbird algorithm (AOAHA). The proposed AOAHA was tested on 23 benchmark functions and compared with the original algorithm and other recent optimization algorithms such as supply-demand-based optimization (SDO), wild horse optimizer (WHO), and tunicate swarm algorithm (TSA). The proposed algorithm was applied to obtain accurate models for solar cell systems, which are the basis of solar power plants, in order to increase their efficiency, thus increasing the efficiency of the whole system. The experiments were carried out on two important models - the static and dynamic models—so that the proposed model would be more representative of real systems. Two applications for static models have been proposed: In the first application, the AOAHA satisfies the best root-mean-square values (0.0009825181). In the second application, the performance of the AOAHA is satisfied in all variable irradiance for the system. The results were evaluated in more than one way, taking into account the comparison with other modern and powerful optimization techniques. Improvement showed its potential through its satisfactory results in the tests that were applied to it.
\end{abstract}

Keywords: permanent energy; benchmark function; static and dynamic models; optimization; AHA; AOAHA

\section{Introduction}

The remarkable development of communication systems and easy access to information are the main factors in the development of artificial intelligence algorithms that, in turn, look at the utilization of these data and produce results that help improve the performance of multiple systems [1]. This improvement has had the effect of increasing the efficiency of these systems, thereby increasing the economic return and guiding the vision for the future. Energy sources are some of the most important systems that researchers have been concerned with developing and making the best use of, because of their economic and strategic value to the whole world. Therefore, this has had a severe impact in increasing the search space around the application of artificial intelligence algorithms to obtain ideal models for these systems, so that developers can study the performance of these systems in the laboratory and, thus, save on high manufacturing costs [2]. 
Modeling of solar systems is one of the contemporary research topics, although it is not a modern research idea, emphasizing its importance, as well as the importance of the improvement algorithms that have begun to intervene in many topics, changing old views on them [3-5].

If we consider photovoltaic (PV) models, there are many studies that have been presented in the past and recently. The most popular PV models are the dynamic and static PV models. The static PV models are based on equivalent circuits containing resistance and diodes [6], because the characteristics of PV cells are similar to those of the semiconductor $\mathrm{P}-\mathrm{N}$ junction (diode) [7]. The popular static models are the single-diode model (SDM), double-diode model (DDM), and three-diode model (TDM). From the names of these models, it is clear that they are distinguished by the number of diodes in the model. The SDM has one diode to represent the diffusion current in the P-N junction [8-10], a resistance connected in series with the diode to represent the total resistance of the semiconductor material at the neutral region, and a resistance connected in parallel with the diode to represent the total current leakage resistance across the $\mathrm{P}-\mathrm{N}$ junction of the solar cell. The DDM has two diodes, one series resistance, and one parallel resistance; the second diode represents the recombination effect in the $\mathrm{P}-\mathrm{N}$ junction [11-14]. The TDM has three diodes, one series resistance, and one parallel resistance; the third diode represents the effect of leakage current and grain boundaries [15-17]. The total estimated parameters for the SDM, DDM, and TDM are five, seven, and nine parameters, respectively. Although increasing the number of diodes has an effect of increasing the model's accuracy, the model's complexity is also increased. Dynamic PV models have been developed to represent the effect of switching and load variation, along with the connection between the PV system and the load. The two most popular PV dynamic models are transfer function models: the integral order model (IOM), and the fractional order model (FOM) [18,19].

As for the application of optimization algorithms to obtain an accurate PV model, many previous studies have been carried out for this purpose [20]. The rapid development of population-based optimization algorithms has enhanced this research area, due to their being meta-heuristic and derivative-free algorithms, unlike greedy algorithms-which solve problems locally in each step, thus depending on finding the best solution in each step based on the previous step-and convex algorithms (e.g., gradient descent algorithms) that depend on the convex function. Therefore, population-based algorithms are suitable for complex and nonlinear functions such as the objective functions of PV models [20,21]. The main challenge in the estimation of PV models' parameters is the nonlinear characteristics of the PV systems, requiring a robust optimization algorithm for this optimization problem. A review of the recent work in this area was presented in [21]. In this study, a collection of recent work on the estimation of PV parameters is discussed. Approximately 29 optimization algorithms are reviewed, such as ABC, MPSO, SSA, and ITLBO. In each reference, the main points discussed are the PV model type, the different applications and case studies proposed, the evaluation methods, and the obtained results. The TDM has seen many applications in the literature, due to its efficiency in representing PV modules such as polycrystalline MSX-60, monocrystalline CS6K-280M and multicrystalline KC200GT. Some studies have applied optimization algorithms to estimate all nine parameters of the TDM, while others tried to estimate two parameters analytically and the rest using optimization algorithms [22]. In this work, the proposed optimization algorithm was used to estimate all nine parameters of the TDM, in order to check and compare its performance with other optimization algorithms through such complex problems.

Different modified algorithms have been proposed in studies such as [23-26]. The AHA is one of the recently proposed algorithms, inspired by the special flight abilities of hummingbirds and their intelligent foraging strategies [27]. The AOAHA is proposed as an enhanced version of the AHA using an adaptive method. The main advantage of the adaptation technique is that the enhancement technique is employed when the algorithm fails to find good solutions. Therefore, the proposed AOAHA improves the performance of the original algorithm by increasing the exploration and exploitation 
balance, thus decreasing the probability of local optima problems. The AOAHA was tested through benchmark functions and applied to estimate the parameters of the static TDM and dynamic IOM and FOM through several applications. The obtained results were analyzed and compared with an original algorithm and other recent algorithms through different evaluation methods.

The main contributions of this paper can be described as follows:

- A novel enhanced algorithm (AOAHA) has been proposed and tested through unimodal, multimodal, and composite benchmark functions, totaling 23 benchmark, functions,

- The enhancement was based on an adaptive opposition approach that suggests whether or not to use an opposition-based learning (OBL) method;

- AOAHA was applied to estimate accurate PV models with consideration of a complex optimization problem, due to the nonlinearities in the PV system's behavior;

- The estimated models and the algorithm behavior were evaluated through different evaluation methods, such as RMSE, absolute error statistical analysis, and algorithm convergence curves;

- The proposed algorithm gives better results than the original and other recent algorithms, both in the benchmark functions and in the real PV application. The enhancement approach increased the exploration and exploitation balance of the original algorithm, as well as its probability of avoiding local optima problems.

The rest of this paper is arranged as follows:

Section 2 presents the PV models (static and dynamic). The proposed AOAHA is discussed in Section 3. The obtained results and their analysis are discussed in Section 4. Section 5 presents the conclusions.

\section{PV Models (Static and Dynamic)}

In this section, the static and dynamic PV models are presented. From the static PV models, the TDM was selected. The main dynamic models (IOM and FOM) are also discussed.

\subsection{Static TDM}

The TDM has three diodes connected in parallel with one another, a resistance connected in series with the group of diodes to represent the total semiconductor material at neutral regions resistance, a resistance connected in parallel with the group of diodes to represent the total current leakage resistance across the $\mathrm{P}-\mathrm{N}$ junction of the solar cell, and a current source connected in parallel with the group of diodes to represent the photo generated, as shown in Figure 1. The TDM has nine parameters, if $x$ is considered a vector of model parameters $x=\left[x_{1}, x_{2}, x_{3}, x_{4}, x_{5}, x_{6}, x_{7}, x_{8}, x_{9}\right]$ equivalent to $\left[R_{s}, R_{s h}, I_{p h}, I_{s 1}, I_{s 2}\right.$, $\left.I_{s 3}, \eta_{1}, \eta_{2}, \eta_{3}\right]$. The TDM is described by Equations (1) and (2). The objective function of the TDM is described by Equation (3).

$$
I=I_{p h}-I_{D 1}-I_{D 2}-I_{D 3}-I_{s h}
$$

where $I$ is the real PV system output current, $I_{p h}$ is a current source representing the generated current from the photons, $I_{D 1}, I_{D 2}$, and $I_{D 2}$ represent the current of the first, second, and third diodes, respectively, and $I_{s h}$ is the current of shunt resistance.

$$
\begin{gathered}
I=I_{p h}-I_{s 1}\left[\exp \left(\frac{q\left(V+R_{s} \cdot I\right)}{\eta_{1} \cdot K \cdot T}\right)-1\right] \\
-I_{s 2}\left[\exp \left(\frac{q\left(V+R_{s} \cdot I\right)}{\eta_{2} \cdot K \cdot T}\right)-1\right]-I_{s 3}\left[\exp \left(\frac{q\left(V+R_{s} \cdot I\right)}{\eta_{3} \cdot K \cdot T}\right)-1\right]-\frac{V+R_{s} \cdot I}{R_{s h}}
\end{gathered}
$$

where $V$ is the PV system output voltage, $R_{S}$ and $R_{s h}$ are the series and shunt resistance, respectively, $\eta_{1}, \eta_{2}$, and $\eta_{3}$ are the ideality factor of the first, second, and third diodes, 
respectively, $K$ is a constant of $=1.380 \times 10^{-23}(\mathrm{~J} / \mathrm{Ko})$, and $q$ is a constant of $1.602 \times 10^{-19}$ (C) coulombs.

$$
\begin{gathered}
F_{T D}(V, I, X)=I-X_{3}-X_{4}\left[\exp \left(\frac{q\left(V+R_{s} \cdot I\right)}{X_{7} \cdot K \cdot T}\right)-1\right] \\
-X_{5}\left[\exp \left(\frac{q\left(V+R_{s} \cdot I\right)}{X_{8} \cdot K \cdot T}\right)-1\right]-X_{6}\left[\exp \left(\frac{q\left(V+R_{s} \cdot I\right)}{X_{9} \cdot K \cdot T}\right)-1\right]-\frac{V+X_{1} \cdot I}{X_{2}}
\end{gathered}
$$

where $F_{T D}$ is the objective function of the TDM.

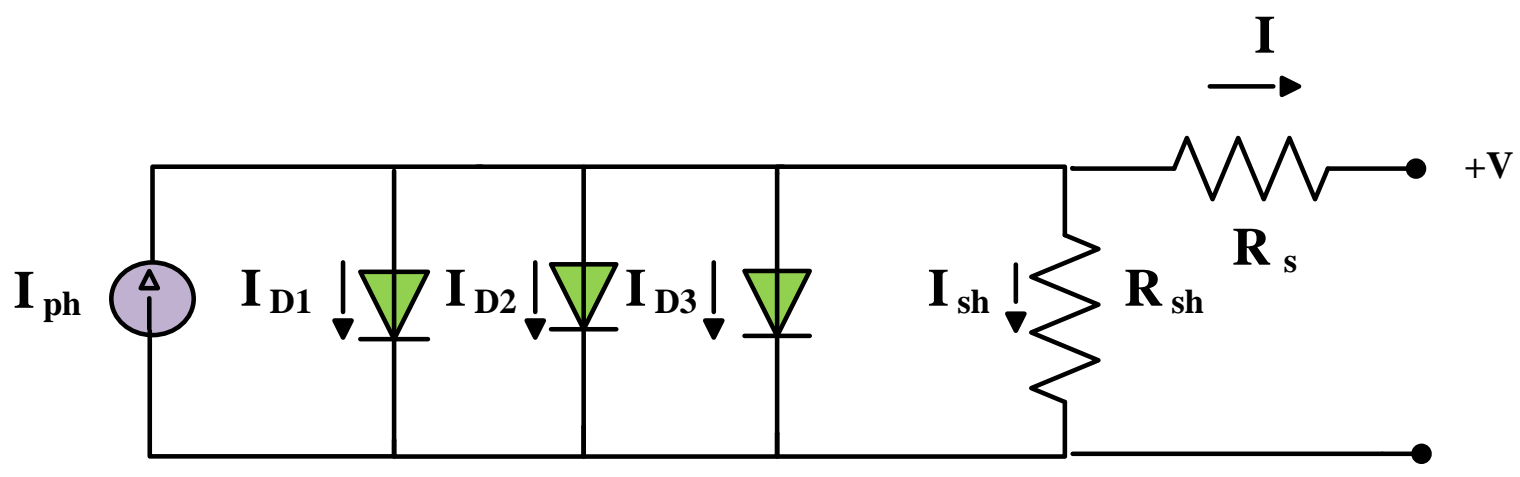

Figure 1. TDM.

\subsection{Dynamic PV Model}

Dynamic PV models have been proposed in the literature to represent the effects of load and switching, as well as the connection between the load and the PV source. The two main models are the integral and fractional order dynamic PV models.

The integral order model (IOM) is a second-order transfer function model. The IOM consists of two parts-one for static and the other for dynamic - as shown in Figure 2, and consists of the following:

- $\quad \mathrm{V}_{\mathrm{oc}}$ : Constant voltage source (static part);

- $\quad R_{S}$ : Series resistance to represent the static model (static part);

- $\quad$ C: Capacitor for junction capacitance (dynamic part);

- $\quad \mathrm{R}_{\mathrm{c}}$ : Resistance for conductance (dynamic part);

- L: The connected cables' inductance is represented by the coil inductance (dynamic part);

- $\quad \mathrm{R}_{\mathrm{L}}$ : Resistance to represent the load (dynamic part).
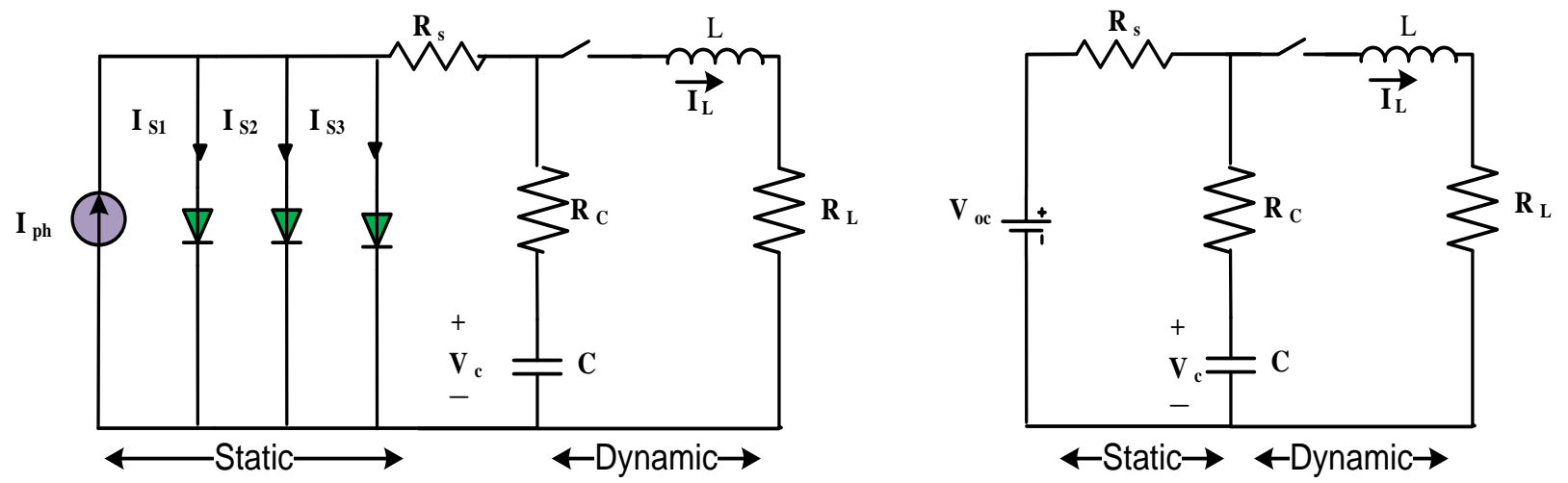

Figure 2. Integral order model.

There are a total of three estimated parameters for the IOM $\left(R_{c}, C\right.$, and $\left.L\right)$; the IOM is represented by Equations (4) and (5).

$$
\mathrm{i}_{\mathrm{L}}(\mathrm{s})=\frac{\mathrm{V}_{\mathrm{oc}}}{\mathrm{s}} \frac{\mathrm{a}_{11}\left(\mathrm{~s}+\mathrm{b}_{1}\right)+\mathrm{b}_{2}\left(\mathrm{~s}-\mathrm{a}_{11}\right)}{\left(\mathrm{s}-\mathrm{a}_{22}\right)\left(\mathrm{s}-\mathrm{a}_{11}\right)-\mathrm{a}_{12} \mathrm{a}_{21}}
$$




$$
\begin{gathered}
\left(\begin{array}{ll}
\mathrm{a}_{11} & \mathrm{a}_{12} \\
\mathrm{a}_{21} & \mathrm{a}_{22}
\end{array}\right)=\left(\begin{array}{c}
\frac{-1}{\mathrm{C}\left(\mathrm{R}_{\mathrm{c}}+\mathrm{R}_{\mathrm{s}}\right)} \frac{-\mathrm{R}_{\mathrm{S}}}{\mathrm{C}\left(\mathrm{R}_{\mathrm{c}}+\mathrm{R}_{\mathrm{s}}\right)} \\
\frac{\mathrm{R}_{\mathrm{S}}}{\mathrm{L}\left(\mathrm{R}_{\mathrm{c}}+\mathrm{R}_{\mathrm{s}}\right)} \frac{-\left[\mathrm{R}_{\mathrm{L}} \mathrm{R}_{\mathrm{c}}+\mathrm{R}_{\mathrm{s}} \mathrm{R}_{\mathrm{c}}+\mathrm{R}_{\mathrm{L}} \mathrm{R}_{\mathrm{s}}\right]}{\mathrm{L}\left(\mathrm{R}_{\mathrm{c}}+\mathrm{R}_{\mathrm{s}}\right)}
\end{array}\right) \\
\left(\begin{array}{c}
\mathrm{b}_{1} \\
\mathrm{~b}_{2}
\end{array}\right)=\left(\begin{array}{c}
\frac{1}{\mathrm{C}\left(\mathrm{R}_{\mathrm{c}}+\mathrm{R}_{\mathrm{s}}\right)} \\
\frac{\mathrm{R}_{\mathrm{c}}}{\mathrm{L}\left(\mathrm{R}_{\mathrm{c}}+\mathrm{R}_{\mathrm{s}}\right)}
\end{array}\right)
\end{gathered}
$$

In transfer function models, the fractional orders are used to represent some components that cannot be represented though the IOM. The FOM can represent the fractional capacitor and capacitance and the fractional inductance, as shown in Figure 3. The fractional order of capacitance and inductance are represented by $\alpha$ and $\beta$, respectively. The total number of FOM parameters is five $\left(R_{c}, C, L, \alpha\right.$, and $\left.\beta\right)$; the FOM is represented by Equations (6) and (7).

$$
\begin{gathered}
\mathrm{i}_{\mathrm{L}}(\mathrm{s})=\frac{\mathrm{V}_{\mathrm{oc}}}{\mathrm{s}} \frac{\mathrm{a}_{11}\left(\mathrm{~s}^{\alpha}+\mathrm{b}_{1}\right)+\mathrm{b}_{2}\left(\mathrm{~s}^{\alpha}-\mathrm{a}_{11}\right)}{\left(\mathrm{s}^{\beta}-\mathrm{a}_{22}\right)\left(\mathrm{s}^{\alpha}-\mathrm{a}_{11}\right)-\mathrm{a}_{12} \mathrm{a}_{21}} \\
\left(\begin{array}{c}
\mathrm{a}_{11} \mathrm{a}_{12} \\
\mathrm{a}_{21} \mathrm{a}_{22}
\end{array}\right)=\left(\begin{array}{c}
\frac{-1}{\mathrm{C}_{\alpha}\left(\mathrm{R}_{\mathrm{c}}+\mathrm{R}_{\mathrm{s}}\right)} \frac{-\mathrm{R}_{\mathrm{S}}}{\mathrm{C}_{\alpha}\left(\mathrm{R}_{\mathrm{c}}+\mathrm{R}_{\mathrm{s}}\right)} \\
\frac{\mathrm{R}_{\mathrm{S}}}{\mathrm{L}_{\beta}\left(\mathrm{R}_{\mathrm{c}}+\mathrm{R}_{\mathrm{s}}\right)} \frac{-\left[\mathrm{R}_{\mathrm{L}} \mathrm{R}_{\mathrm{c}}+\mathrm{R}_{\mathrm{s}} \mathrm{R}_{\mathrm{c}}+\mathrm{R}_{\mathrm{L}} \mathrm{R}_{\mathrm{s}}\right]}{\mathrm{L}_{\beta}\left(\mathrm{R}_{\mathrm{c}}+\mathrm{R}_{\mathrm{s}}\right)}
\end{array}\right) \\
\left(\begin{array}{c}
\mathrm{b}_{1} \\
\mathrm{~b}_{2}
\end{array}\right)=\left(\begin{array}{c}
\frac{1}{\mathrm{C}_{\alpha}\left(\mathrm{R}_{\mathrm{c}}+\mathrm{R}_{\mathrm{s}}\right)} \\
\frac{\mathrm{R}_{\mathrm{c}}}{\mathrm{L}_{\beta}\left(\mathrm{R}_{\mathrm{c}}+\mathrm{R}_{\mathrm{s}}\right)}
\end{array}\right)
\end{gathered}
$$

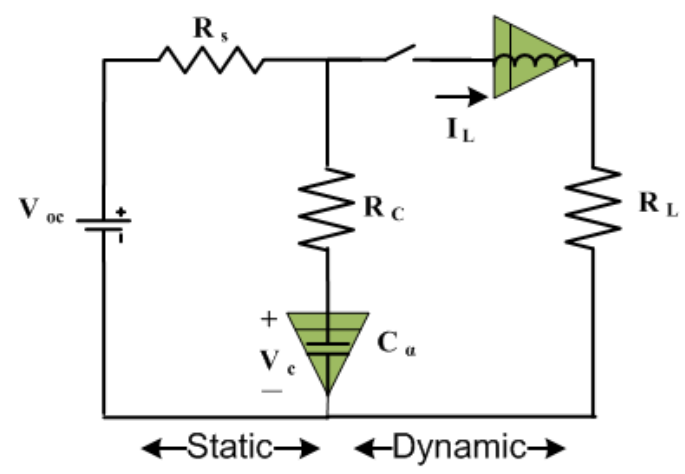

Figure 3. Fractional order model.

\section{The Proposed Optimization Methodology}

This section briefly defines the basics of the artificial hummingbird algorithm (AHA). Then, the process of the proposed adaptive opposition artificial hummingbird algorithm (AOAHA) is described.

\subsection{Artificial Hummingbird Algorithm (AHA)}

The AHA is an optimization technique inspired by the foraging and flight of hummingbirds, as presented in [27]. The three main models of this algorithm are presented as follows:

\section{(a) Guided foraging}

In this foraging model, three flight behaviors are used in foraging (omnidirectional, diagonal, and axial flight). Figure 4 presents these three flight behaviors in 3D space. The equation simulating this guided foraging and a candidate food source can be obtained as follows:

$$
v_{i}(t+1)=x_{i, t a}(t)+h \cdot b \cdot\left(x_{i}(t)-X_{i, t a}(t)\right) h \sim N(0,1)
$$

where $x_{i, t a}(t)$ represents the position of the target food source, $h$ denotes the guided factor, and $x_{i}(t)$ is the position of the $i$ th food source at time $t$. 


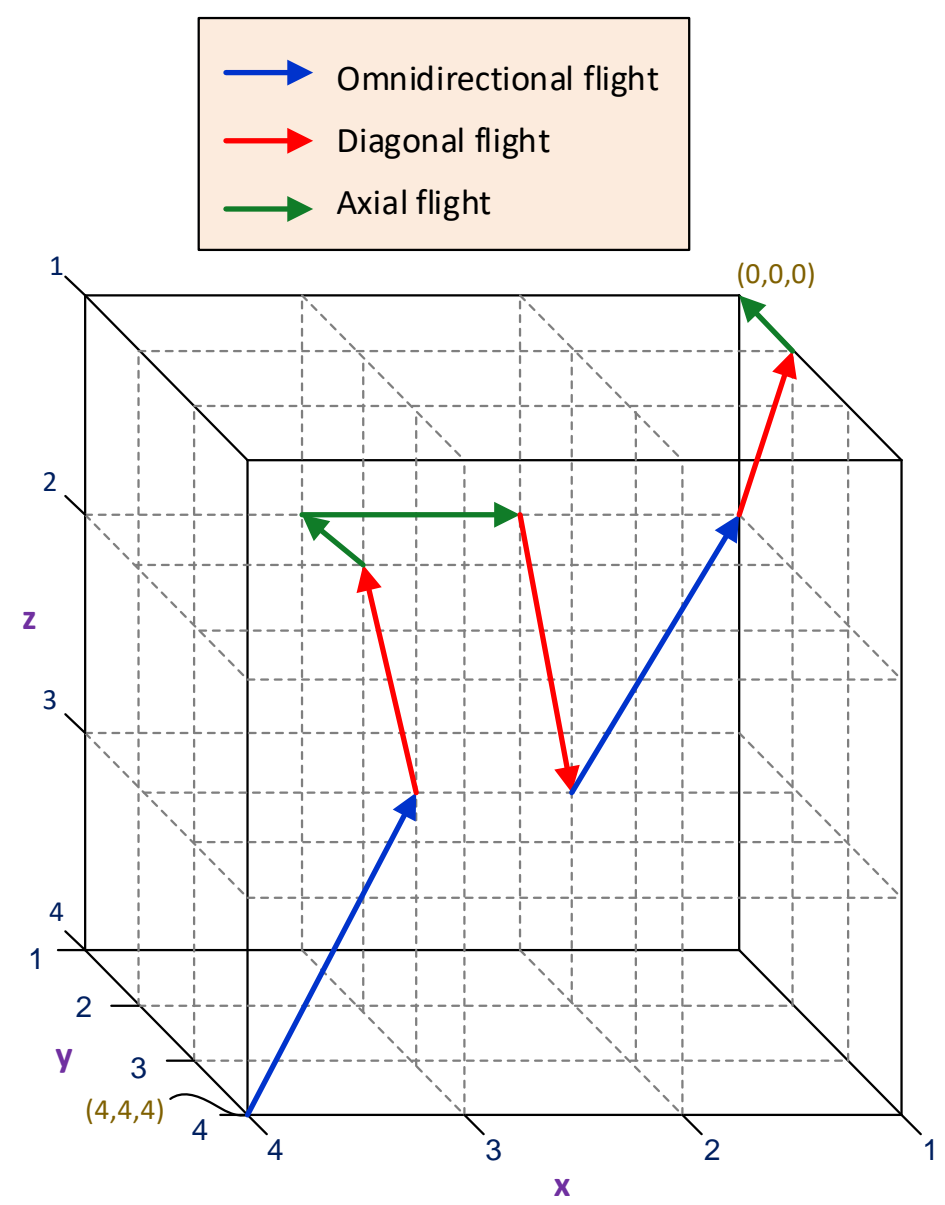

Figure 4. Three flight behaviors in 3D space.

The position update of the $i$ th food source is as follows:

$$
x_{A i}(t)=\left\{\begin{array}{c}
x_{i}(t) \quad f\left(x_{i}(t)\right) \leq f\left(v_{i}(t+1)\right) \\
v_{i}(t+1) f\left(x_{i}(t)\right)>f\left(v_{i}(t+1)\right)
\end{array}\right.
$$

where $f\left(x_{i}(t)\right)$ and $f\left(v_{i}(t+1)\right)$ are the value of function fitness for $x_{i}(t)$ and $v_{i}(t+1)$, respectively. (b) Territorial foraging

The following equation represents the local search of hummingbirds in the territorial foraging strategy:

$$
v_{i}(t+1)=x_{i}(t)+g \cdot b \cdot\left(x_{i}(t)\right) g \sim N(0,1)
$$

where $g$ denotes the territorial factor.

\section{(c) Migration foraging}

The mathematical equation for the migration foraging of a hummingbird is presented as follows:

$$
x_{w o r}(t+1)=l b+r \cdot(u b-l b)
$$

where $x_{w o r}$ represents the source of food with worst population rate of nectar refilling, $r$ is a random factor, and $u b$ and $l b$ are the upper and lower limit ranges, respectively.

\subsection{Adaptive Opposition Artificial Hummingbird Algorithm (AOAHA)}

It is suggested to use an adaptive approach to select whether or not to use an opposition-based learning (OBL) method. This idea is used to further improve the exploration ability; furthermore, it ensures the maximization of the exploitation stage by replacing one random search agent with the best one in the updated position [28]. 


\section{(a) Opposition-based learning}

OBL uses the position $x o_{i}(t)$ in the search space, which is the accurate opposite of the position $x_{i}(t)$ of the $i$ th food source, and compares it to update the position of the next iterations. This method helps to reduce the chances of being trapped in the local optima with developed convergence. The $x_{o i}(t)$ position is calculated as follows:

$$
x o_{i}^{j}(t)=\min \left(x_{i}(t)\right)+\max \left(x_{i}(t)-x_{i}^{j}(t)\right)
$$

where $j=1,2, \ldots, \mathrm{d}$. $\mathrm{d}$ denotes the dimension.

The position of the $i$ th food source is as follows:

$$
x_{i}(t)=\left\{\begin{array}{cl}
x o_{i}(t) & f\left(x o_{i}(t)\right) \leq f\left(x_{i}(t)\right) \\
x_{i}(t) & f\left(x o_{i}(t)\right)>f\left(x_{i}(t)\right)
\end{array}\right.
$$

where $f\left(x o_{i}(t)\right)$ is the value of function fitness for $x o_{i}(t)$.

\section{(b) Adaptive decision strategy}

The adaptive decision helps to further improve exploration through the OBL when needed. Finally, the position updates using the adaptive decision strategy of AOAHA, which is shown as follows:

$$
x_{i}(t+1)=\left\{\begin{array}{cl}
x_{A i}(t) & f\left(x_{A i}(t)\right) \leq f\left(x_{i}(t)\right) \\
x_{i}(t) & f\left(x_{A i}(t)\right)>f\left(x_{i}(t)\right)
\end{array}\right.
$$

\section{Results}

\subsection{The Performance of the AOAHA}

The proficiency and performance of the proposed AOAHA technique were evaluated based on several benchmark functions, using he statistical measurements such as best values, mean values, median values, worst values, and standard deviation (STD) for the best solutions acquired by the AOAHA and the other state-of-the-art optimization algorithms. The results achieved with the proposed AOAHA were compared with three recent algorithms - supply-demand-based optimization (SDO) [29], wild horse optimizer (WHO) [30], and tunicate swarm algorithm (TSA) [31] —and the original artificial hummingbird algorithm (AHA). Figure 5 shows the qualitative metrics on F1, F2, F3, F5, F6, F8, F10, F12, F15, F18, and F22, with 2D views of the functions, convergence curve, average fitness history, and search history. 
F1
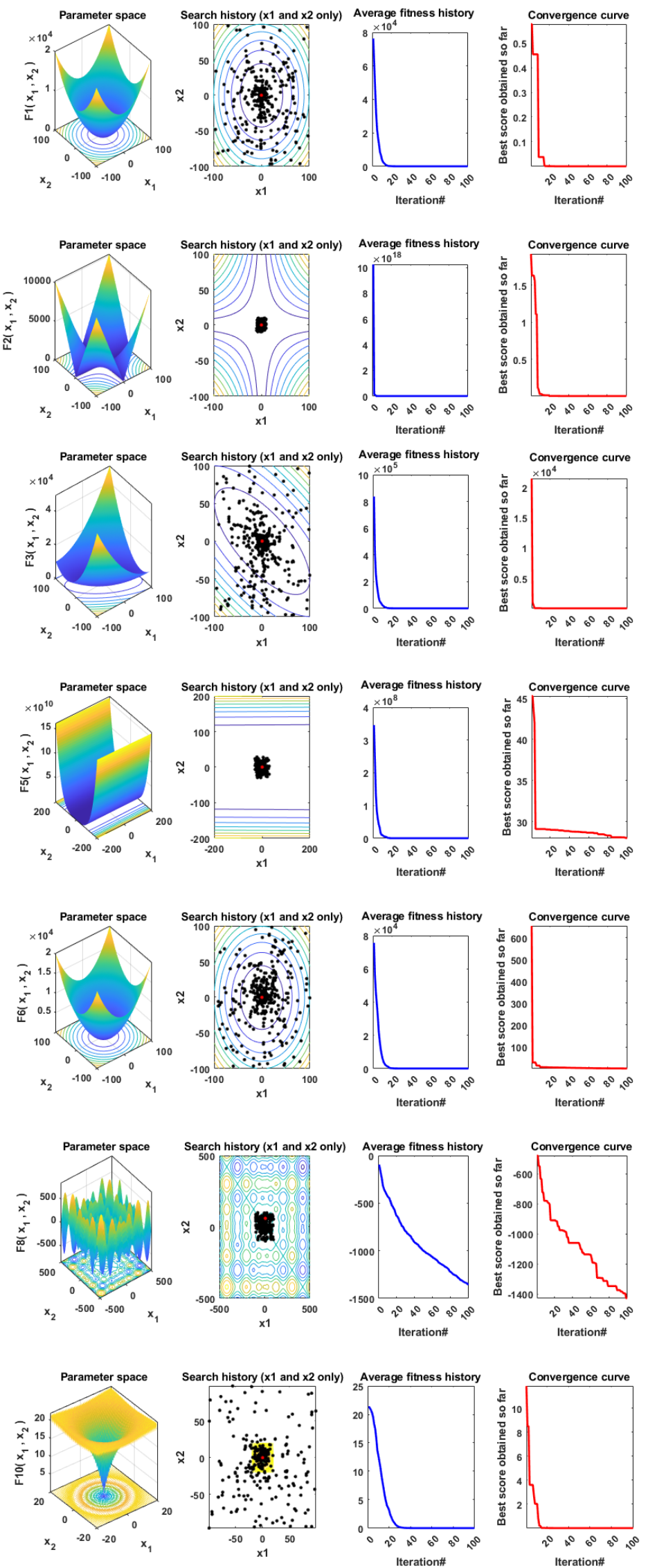

Figure 5. Cont. 

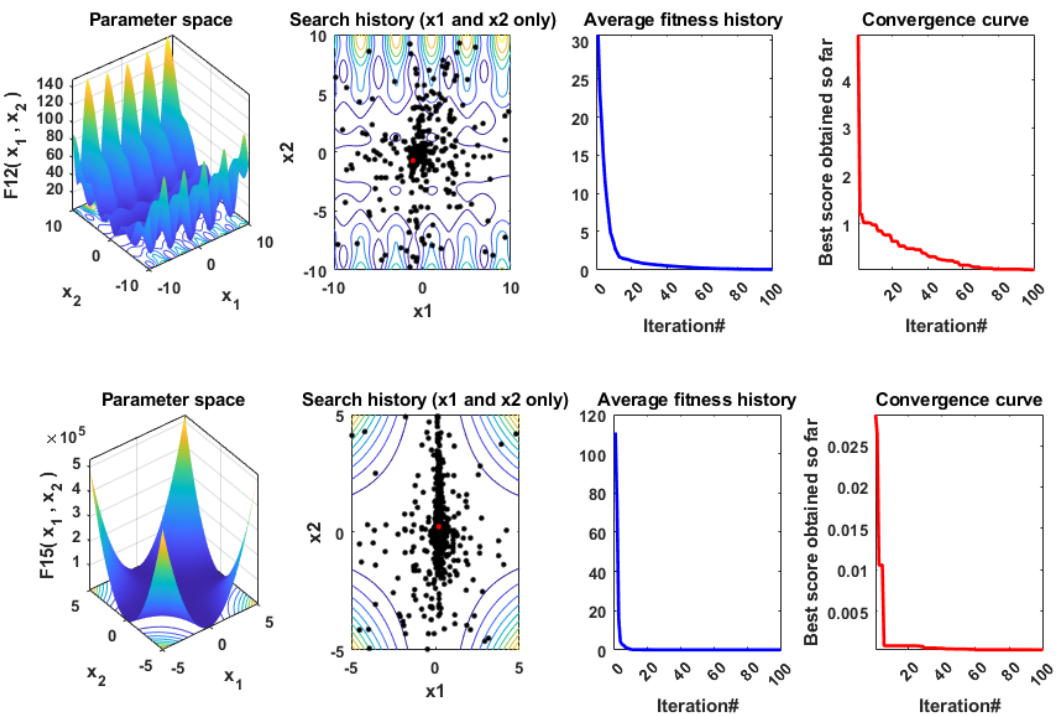

F18
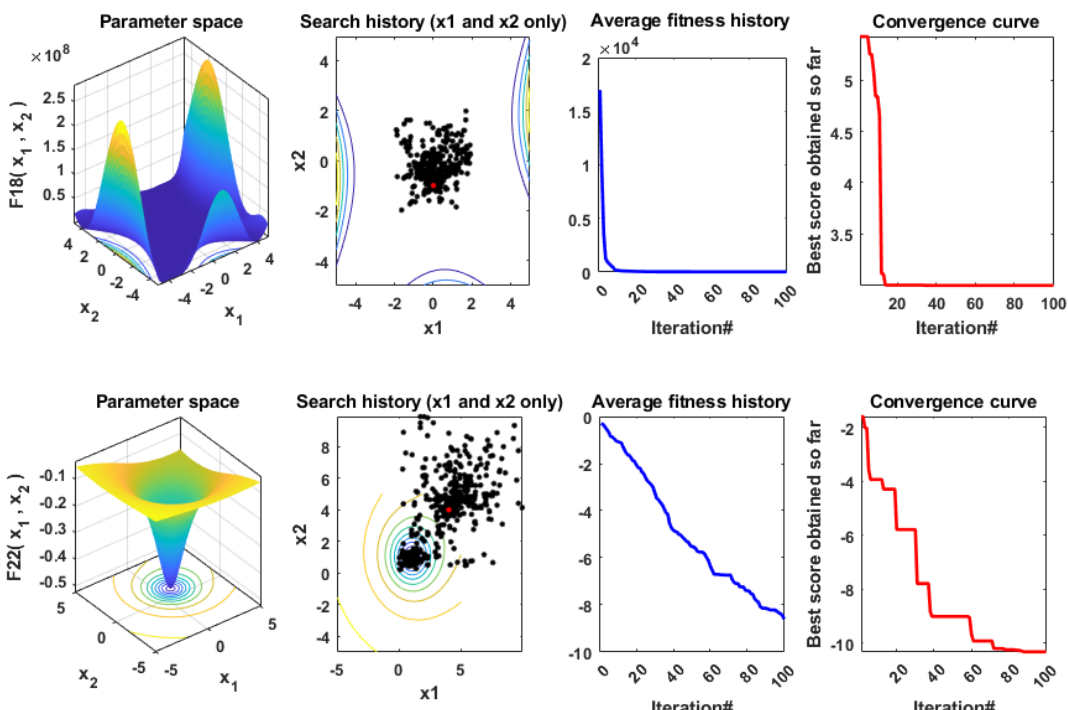

Figure 5. Qualitative metrics of 12 benchmark functions: 2D views of the functions, search history, average fitness history, and convergence curve using the AOAHA technique.

Tables 1-3 show the statistical results of the AOAHA and other recent algorithms when applied for the unimodal benchmark functions, multimodal benchmark functions, and composite benchmark functions, respectively. The best values were achieved with the AOAHA, AHA, SDO, WHO, and TSA algorithms, as shown in bold. It can clearly be seen that the AOAHA technique attains the best solutions for most of these benchmark functions. The convergence curves of these algorithms for these functions are presented in Figure 6, while the boxplots for each algorithm for these functions are presented in Figure 7. From those figures, it is clear that the AOAHA algorithm reaches a stable point for all functions, and the boxplots of the AOAHA algorithm are very narrow and stable for most functions compared to the other algorithms. 
Table 1. Results of unimodal benchmark functions.

\begin{tabular}{|c|c|c|c|c|c|c|}
\hline & Function & АОАНА & AHA & SDO & WHO & TSA \\
\hline \multirow{6}{*}{ F1 } & Best & $1.29 \times 10^{-66}$ & $3.01 \times 10^{-66}$ & $1.39 \times 10^{-55}$ & $5.08 \times 10^{-21}$ & $3.79 \times 10^{-8}$ \\
\hline & Mean & $9.14 \times 10^{-56}$ & $3.87 \times 10^{-53}$ & $1.49 \times 10^{-51}$ & $2.13 \times 10^{-18}$ & $4.64 \times 10^{-7}$ \\
\hline & Median & $4.31 \times 10^{-59}$ & $3.32 \times 10^{-59}$ & $3.74 \times 10^{-54}$ & $6.47 \times 10^{-19}$ & $1.17 \times 10^{-7}$ \\
\hline & Worst & $1.54 \times 10^{-54}$ & $7.66 \times 10^{-52}$ & $8.43 \times 10^{-51}$ & $8.56 \times 10^{-18}$ & $4.09 \times 10^{-6}$ \\
\hline & STD & $3.48 \times 10^{-55}$ & $1.71 \times 10^{-52}$ & $2.99 \times 10^{-51}$ & $2.98 \times 10^{-18}$ & $1.15 \times 10^{-6}$ \\
\hline & Best & $6.71 \times 10^{-35}$ & $4.74 \times 10^{-34}$ & $1.83 \times 10^{-29}$ & $4.13 \times 10^{-13}$ & $2.44 \times 10^{-6}$ \\
\hline \multirow{4}{*}{ F2 } & Mean & $5.66 \times 10^{-29}$ & $1.07 \times 10^{-29}$ & $3.76 \times 10^{-25}$ & $1.3 \times 10^{-10}$ & $1.9 \times 10^{-5}$ \\
\hline & Median & $1.12 \times 10^{-30}$ & $3.11 \times 10^{-31}$ & $1.13 \times 10^{-26}$ & $5.29 \times 10^{-11}$ & $1.86 \times 10^{-5}$ \\
\hline & Worst & $4.08 \times 10^{-28}$ & $9.48 \times 10^{-29}$ & $3.98 \times 10^{-24}$ & $6.34 \times 10^{-10}$ & $3.68 \times 10^{-5}$ \\
\hline & STD & $1.25 \times 10^{-28}$ & $2.51 \times 10^{-29}$ & $9.1 \times 10^{-25}$ & $1.77 \times 10^{-10}$ & $9.44 \times 10^{-6}$ \\
\hline \multirow{5}{*}{ F3 } & Best & $2.43 \times 10^{-61}$ & $3.15 \times 10^{-61}$ & $6.27 \times 10^{-46}$ & $5.13 \times 10^{-13}$ & 0.027608 \\
\hline & Mean & $1.59 \times 10^{-50}$ & $4.36 \times 10^{-48}$ & $6.91 \times 10^{-34}$ & $1.2 \times 10^{-8}$ & 1.122677 \\
\hline & Median & $3.03 \times 10^{-54}$ & $1.01 \times 10^{-54}$ & $1.4 \times 10^{-39}$ & $6.29 \times 10^{-11}$ & 0.772195 \\
\hline & Worst & $3.06 \times 10^{-49}$ & $6.68 \times 10^{-47}$ & $1.38 \times 10^{-32}$ & $2.3 \times 10^{-7}$ & 3.914695 \\
\hline & STD & $6.82 \times 10^{-50}$ & $1.53 \times 10^{-47}$ & $3.09 \times 10^{-33}$ & $5.14 \times 10^{-8}$ & 1.096313 \\
\hline \multirow{5}{*}{ F4 } & Best & $1.28 \times 10^{-32}$ & $5.07 \times 10^{-29}$ & $1.11 \times 10^{-26}$ & $5.11 \times 10^{-9}$ & 0.67531 \\
\hline & Mean & $3.07 \times 10^{-24}$ & $4.63 \times 10^{-26}$ & $4.52 \times 10^{-23}$ & $3.5 \times 10^{-7}$ & 3.616654 \\
\hline & Median & $5.11 \times 10^{-27}$ & $1.05 \times 10^{-27}$ & $1.14 \times 10^{-23}$ & $1 \times 10^{-7}$ & 3.022253 \\
\hline & Worst & $4.85 \times 10^{-23}$ & $4.23 \times 10^{-25}$ & $1.94 \times 10^{-22}$ & $2.14 \times 10^{-6}$ & 9.361516 \\
\hline & STD & $1.11 \times 10^{-23}$ & $1.02 \times 10^{-25}$ & $6.34 \times 10^{-23}$ & $6.09 \times 10^{-7}$ & 2.343658 \\
\hline \multirow{5}{*}{ F5 } & Best & 26.8806 & 26.40974 & 27.90967 & 26.68451 & 27.18973 \\
\hline & Mean & 27.71771 & 27.5024 & 28.65096 & 37.10656 & 39.01094 \\
\hline & Median & 27.60593 & 27.47815 & 28.74726 & 27.67985 & 28.66203 \\
\hline & Worst & 28.73785 & 28.53304 & 28.98699 & 208.5133 & 239.7785 \\
\hline & STD & 0.597793 & 0.472237 & 0.295026 & 40.37046 & 47.26339 \\
\hline \multirow{5}{*}{ F6 } & Best & 0.037049 & 0.058638 & 0.039957 & 0.013248 & 2.886997 \\
\hline & Mean & 0.449979 & 0.442296 & 2.568541 & 0.064784 & 3.800719 \\
\hline & Median & 0.36532 & 0.393054 & 2.038779 & 0.058665 & 3.736935 \\
\hline & Worst & 1.188272 & 1.029767 & 7.250251 & 0.16971 & 4.850371 \\
\hline & STD & 0.306108 & 0.249876 & 1.852701 & 0.043941 & 0.527851 \\
\hline \multirow{5}{*}{ F7 } & Best & $5.14 \times 10^{-5}$ & $1.47 \times 10^{-5}$ & $8.66 \times 10^{-5}$ & 0.000605 & 0.007604 \\
\hline & Mean & 0.000397 & 0.000346 & 0.002356 & 0.001779 & 0.019206 \\
\hline & Median & 0.000335 & 0.000219 & 0.001136 & 0.001387 & 0.018479 \\
\hline & Worst & 0.001143 & 0.001202 & 0.013813 & 0.004938 & 0.04436 \\
\hline & STD & 0.000298 & 0.000292 & 0.003331 & 0.001255 & 0.007628 \\
\hline
\end{tabular}

The best values obtained are shown in bold.

Table 2. Results of multimodal benchmark functions.

\begin{tabular}{|c|c|c|c|c|c|c|}
\hline & tion & АОАНА & AHA & SDO & WHO & TSA \\
\hline \multirow{5}{*}{ F8 } & Best & -1678.77 & -1724.06 & -1655 & -1807.46 & -1394.45 \\
\hline & Mean & -1551.15 & -1551.13 & -1312.83 & -1721.44 & -1212.82 \\
\hline & Median & -1544.23 & -1562.44 & -1385.86 & -1729.69 & -1232.52 \\
\hline & Worst & -1443.17 & -1364.15 & -598.802 & -1630.81 & -976.635 \\
\hline & STD & 69.17895 & 93.45685 & 294.008 & 54.13894 & 122.0762 \\
\hline \multirow{5}{*}{ F9 } & Best & 0.00 & 0.00 & $4.33 \times 10^{-30}$ & 0.00 & 156.667 \\
\hline & Mean & 0.00 & 0.00 & $1.75 \times 10^{-22}$ & $1.11 \times 10^{-5}$ & 228.0177 \\
\hline & Median & 0.00 & 0.00 & $4.17 \times 10^{-25}$ & $1 \times 10^{-9}$ & 228.634 \\
\hline & Worst & 0.00 & 0.00 & $3.02 \times 10^{-21}$ & 0.000177 & 331.7581 \\
\hline & STD & 0.00 & 0.00 & $6.75 \times 10^{-22}$ & $3.96 \times 10^{-5}$ & 46.40919 \\
\hline \multirow{5}{*}{ F10 } & Best & $8.88 \times 10^{-16}$ & $8.88 \times 10^{-16}$ & $8.88 \times 10^{-16}$ & $8.88 \times 10^{-16}$ & 20.81133 \\
\hline & Mean & $8.88 \times 10^{-16}$ & $8.88 \times 10^{-16}$ & $8.88 \times 10^{-16}$ & 1.003597 & 20.9608 \\
\hline & Median & $8.88 \times 10^{-16}$ & $8.88 \times 10^{-16}$ & $8.88 \times 10^{-16}$ & $7.99 \times 10^{-6}$ & 20.99356 \\
\hline & Worst & $8.88 \times 10^{-16}$ & $8.88 \times 10^{-16}$ & $8.88 \times 10^{-16}$ & 20.01369 & 21.0961 \\
\hline & STD & 0.00 & 0.00 & 0.00 & 4.474524 & 0.091505 \\
\hline
\end{tabular}


Table 2. Cont.

\begin{tabular}{|c|c|c|c|c|c|c|}
\hline & tion & AOAHA & AHA & SDO & WHO & TSA \\
\hline \multirow{5}{*}{ F11 } & Best & 0.00 & 0.00 & 0.00 & 0.00 & $1.3 \times 10^{-9}$ \\
\hline & Mean & 0.00 & 0.00 & 0.00 & $1.83 \times 10^{-16}$ & 0.007018 \\
\hline & Median & 0.00 & 0.00 & 0.00 & 0.00 & $1.44 \times 10^{-8}$ \\
\hline & Worst & 0.00 & 0.00 & 0.00 & $3.66 \times 10^{-15}$ & 0.029126 \\
\hline & STD & 0.00 & 0.00 & 0.00 & $8.19 \times 10^{-16}$ & 0.010243 \\
\hline \multirow{5}{*}{ F12 } & Best & 0.00112 & 0.001029 & 0.001152 & $4.64 \times 10^{-5}$ & 0.374956 \\
\hline & Mean & 0.009553 & 0.008654 & 0.23467 & 0.026544 & 2.805889 \\
\hline & Median & 0.009173 & 0.006918 & 0.067805 & 0.000309 & 2.009833 \\
\hline & Worst & 0.020446 & 0.031416 & 1.492821 & 0.207386 & 7.656863 \\
\hline & STD & 0.005674 & 0.007552 & 0.352063 & 0.056802 & 2.128936 \\
\hline \multirow{5}{*}{ F13 } & Best & 0.433176 & 1.456302 & 0.046216 & 0.011802 & 2.372295 \\
\hline & Mean & 2.155627 & 2.339115 & 1.867552 & 0.173897 & 3.298085 \\
\hline & Median & 2.401709 & 2.436057 & 1.934246 & 0.136817 & 3.22876 \\
\hline & Worst & 2.969199 & 2.969591 & 2.999924 & 0.700833 & 4.16073 \\
\hline & STD & 0.723935 & 0.361111 & 0.961284 & 0.157716 & 0.565835 \\
\hline
\end{tabular}

The best values obtained are shown in bold.

Table 3. Results of composite benchmark functions.

\begin{tabular}{|c|c|c|c|c|c|c|}
\hline & tion & AOAHA & AHA & SDO & WHO & TSA \\
\hline \multirow{5}{*}{ F14 } & Best & 0.998004 & 0.998004 & 0.998004 & 0.998004 & 0.998004 \\
\hline & Mean & 0.998004 & 0.998004 & 3.494696 & 1.097209 & 8.298683 \\
\hline & Median & 0.998004 & 0.998004 & 1.495017 & 0.998004 & 10.76318 \\
\hline & Worst & 0.998004 & 0.998004 & 12.67051 & 2.982105 & 18.30431 \\
\hline & STD & $1.76 \times 10^{-8}$ & $1.03 \times 10^{-9}$ & 3.953203 & 0.443659 & 5.533952 \\
\hline \multirow{5}{*}{ F15 } & Best & 0.000307 & 0.000307 & 0.000307 & 0.000307 & 0.000308 \\
\hline & Mean & 0.000308 & 0.000318 & 0.00067 & 0.000602 & 0.007136 \\
\hline & Median & 0.000308 & 0.000308 & 0.000527 & 0.000593 & 0.000505 \\
\hline & Worst & 0.00032 & 0.000485 & 0.002121 & 0.001223 & 0.031699 \\
\hline & STD & $2.69 \times 10^{-6}$ & $3.95 \times 10^{-5}$ & 0.000473 & 0.000286 & 0.010606 \\
\hline \multirow{5}{*}{ F16 } & Best & -1.03163 & -1.03163 & -1.03163 & -1.03163 & -1.03163 \\
\hline & Mean & -1.03163 & -1.03163 & -1.03005 & -1.03163 & -1.0253 \\
\hline & Median & -1.03163 & -1.03163 & -1.03163 & -1.03163 & -1.03163 \\
\hline & Worst & -1.03163 & -1.03163 & -1.00046 & -1.03163 & -0.99999 \\
\hline & STD & $1.3 \times 10^{-12}$ & $1.18 \times 10^{-12}$ & 0.006966 & $5.09 \times 10^{-17}$ & 0.012981 \\
\hline \multirow{5}{*}{ F17 } & Best & 0.397887 & 0.397887 & 0.397887 & 0.397887 & 0.39789 \\
\hline & Mean & 0.397887 & 0.397887 & 0.397987 & 0.397887 & 0.397927 \\
\hline & Median & 0.397887 & 0.397887 & 0.397887 & 0.397887 & 0.397907 \\
\hline & Worst & 0.397887 & 0.397887 & 0.399795 & 0.397887 & 0.398082 \\
\hline & STD & 0.00 & 0.00 & 0.000426 & 0.00 & $4.53 \times 10^{-5}$ \\
\hline \multirow{5}{*}{ F18 } & Best & 3.00 & 3.00 & 3.00 & 3.00 & 3.000009 \\
\hline & Mean & 3.00 & 3.00 & 3.00 & 3.00 & 8.400078 \\
\hline & Median & 3.00 & 3.00 & 3.00 & 3.00 & 3.000084 \\
\hline & Worst & 3.00 & 3.00 & 3.00 & 3.00 & 84.00001 \\
\hline & STD & $1.77 \times 10^{-15}$ & $1.6 \times 10^{-15}$ & $5.21 \times 10^{-8}$ & $1.13 \times 10^{-15}$ & 18.78799 \\
\hline \multirow{5}{*}{ F19 } & Best & -0.30048 & -0.30048 & -0.30048 & -0.30048 & -0.30048 \\
\hline & Mean & -0.30047 & -0.30047 & -0.2893 & -0.30048 & -0.30048 \\
\hline & Median & -0.30047 & -0.30047 & -0.30038 & -0.30048 & -0.30048 \\
\hline & Worst & -0.30046 & -0.30044 & -0.19165 & -0.30048 & -0.30048 \\
\hline & STD & $4.22 \times 10^{-6}$ & $1.04 \times 10^{-5}$ & 0.026531 & $1.14 \times 10^{-16}$ & $1.14 \times 10^{-16}$ \\
\hline \multirow{5}{*}{ F20 } & Best & -3.322 & -3.322 & -3.322 & -3.322 & -3.32148 \\
\hline & Mean & -3.29227 & -3.30415 & -3.09697 & -3.21756 & -3.07223 \\
\hline & Median & -3.322 & -3.322 & -3.2031 & -3.322 & -3.20118 \\
\hline & Worst & -3.2031 & -3.2031 & -0.89904 & -2.43178 & -0.20816 \\
\hline & STD & 0.052819 & 0.043552 & 0.550986 & 0.239908 & 0.679321 \\
\hline \multirow{5}{*}{ F21 } & Best & -10.1532 & -10.1532 & -10.1532 & -10.1532 & -10.0895 \\
\hline & Mean & -9.89798 & -10.1059 & -8.703 & -9.77706 & -5.89545 \\
\hline & Median & -10.1531 & -10.153 & -10.1532 & -10.1532 & -4.90994 \\
\hline & Worst & -5.0552 & -9.2237 & -4.99677 & -2.63047 & -2.58642 \\
\hline & STD & 1.139873 & 0.207648 & 2.23952 & 1.682133 & 2.775111 \\
\hline \multirow{5}{*}{$\mathrm{F} 22$} & Best & -10.4029 & -10.4029 & -10.4029 & -10.4029 & -10.3637 \\
\hline & Mean & -10.135 & -10.0864 & -8.45822 & -9.75463 & -7.02119 \\
\hline & Median & -10.4029 & -10.4029 & -10.4029 & -10.4029 & -9.8942 \\
\hline & Worst & -5.08767 & -5.08767 & -1.0677 & -2.75193 & -1.82478 \\
\hline & STD & 1.188023 & 1.19136 & 3.128689 & 2.031123 & 3.57071 \\
\hline \multirow{5}{*}{ F23 } & Best & -10.5364 & -10.5364 & -10.5364 & -10.5364 & -10.4599 \\
\hline & Mean & -10.1167 & -10.2621 & -7.90449 & -10.5364 & -5.50502 \\
\hline & Median & -10.5364 & -10.5364 & -10.5357 & -10.5364 & -2.83596 \\
\hline & Worst & -5.12848 & -5.12848 & -3.79083 & -10.5364 & -1.66783 \\
\hline & STD & 1.348528 & 1.208388 & 3.015319 & $1.58 \times 10^{-15}$ & 3.728197 \\
\hline
\end{tabular}



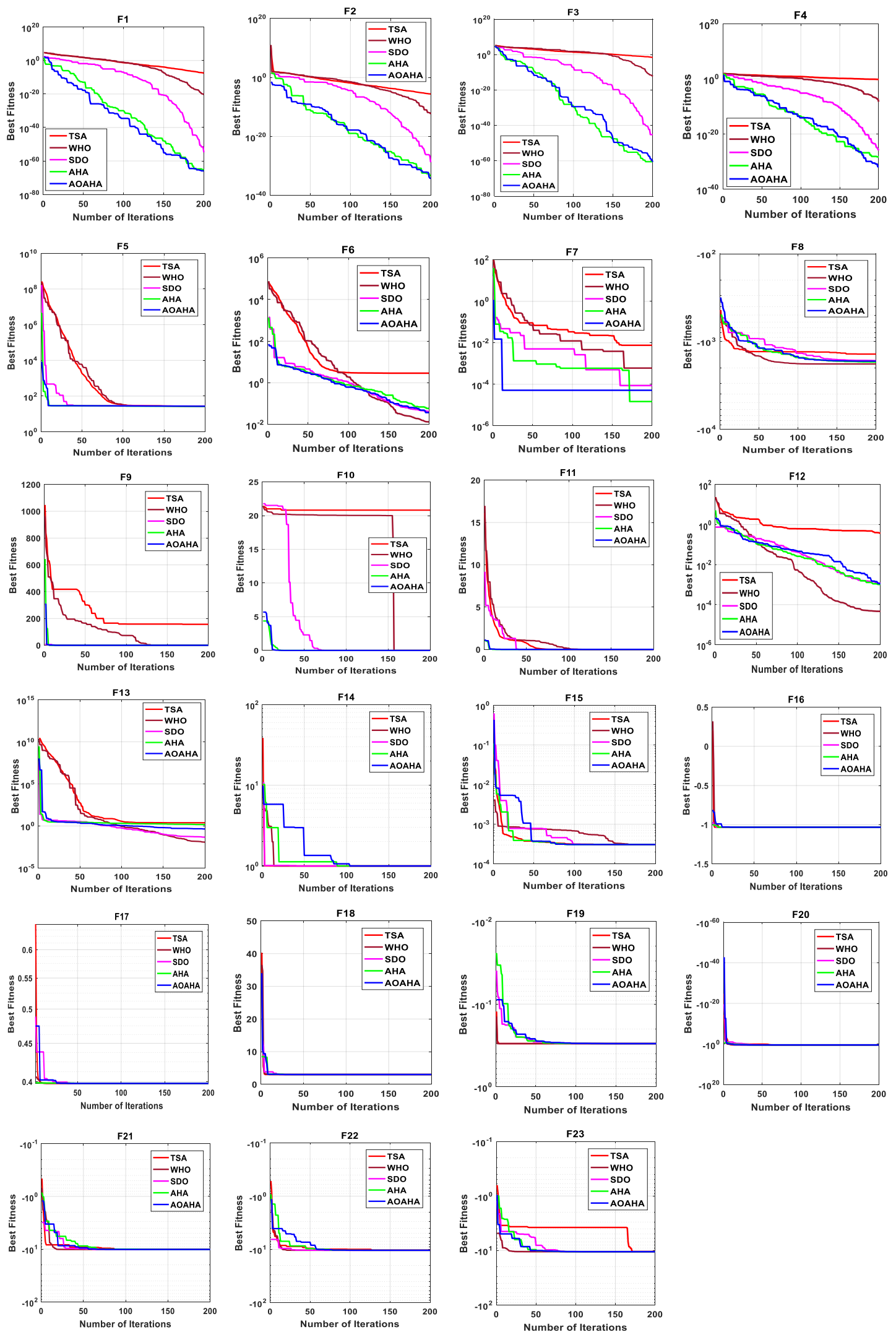

Figure 6. The convergence curves of all algorithms for 23 benchmark functions. 

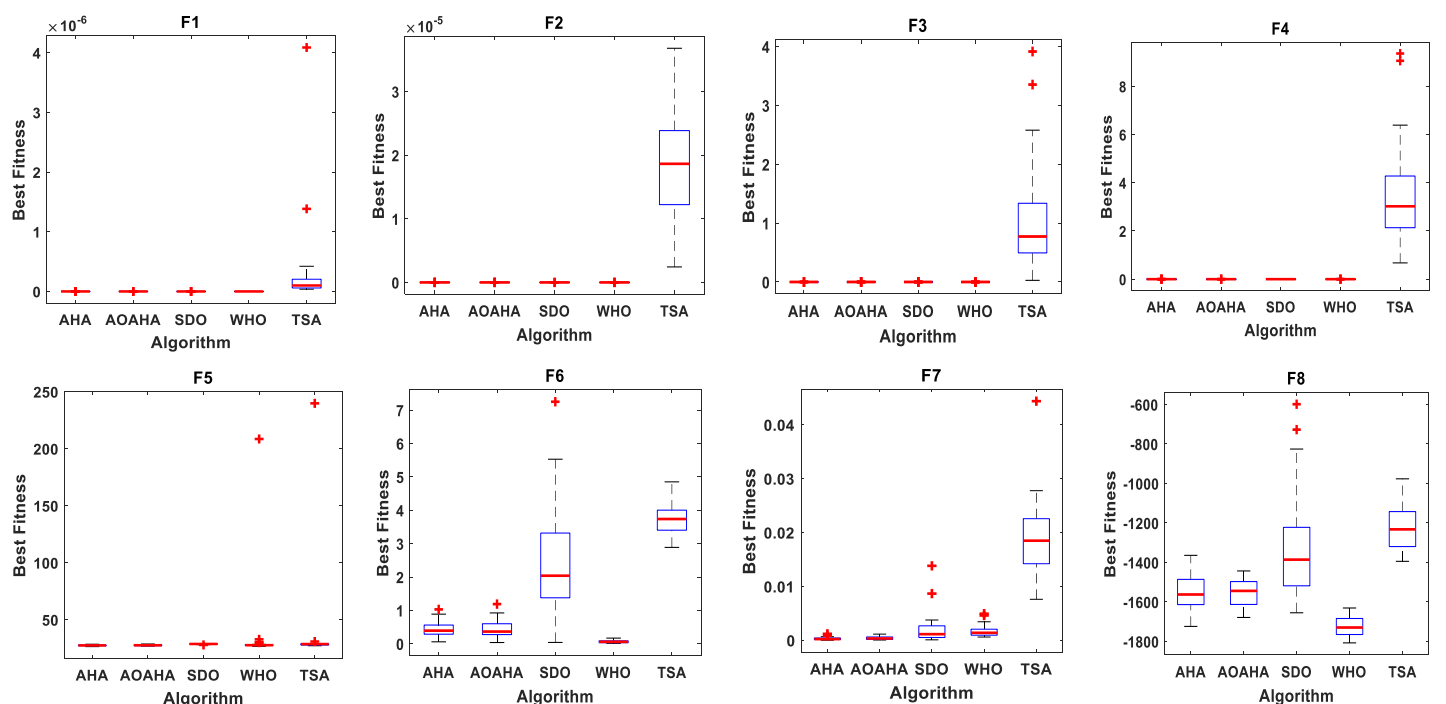

F9

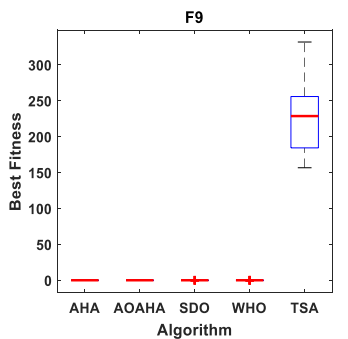

F10

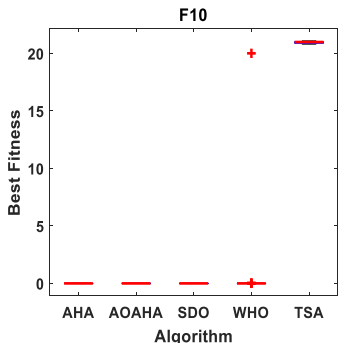

F11

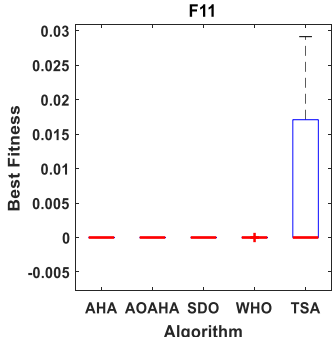

F12

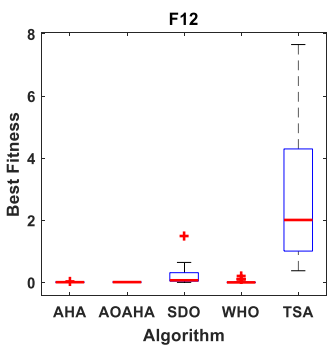

F14
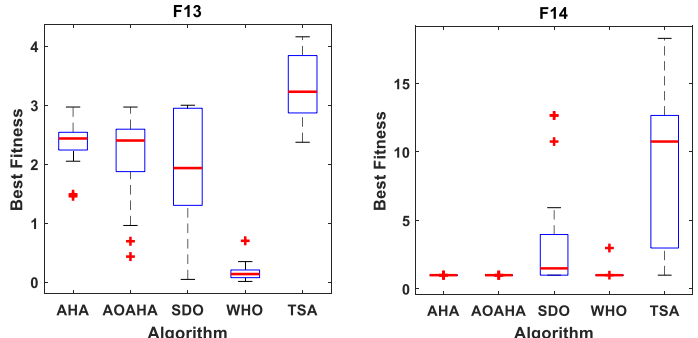

F1

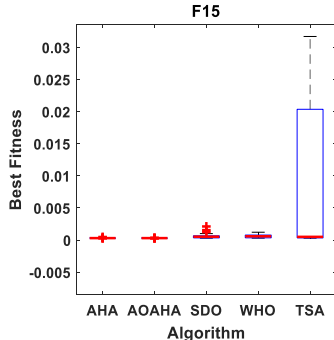

F16

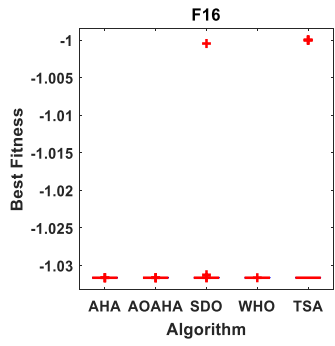

F17

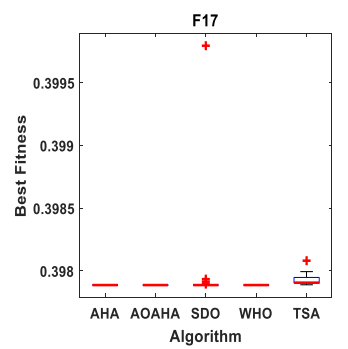

F18
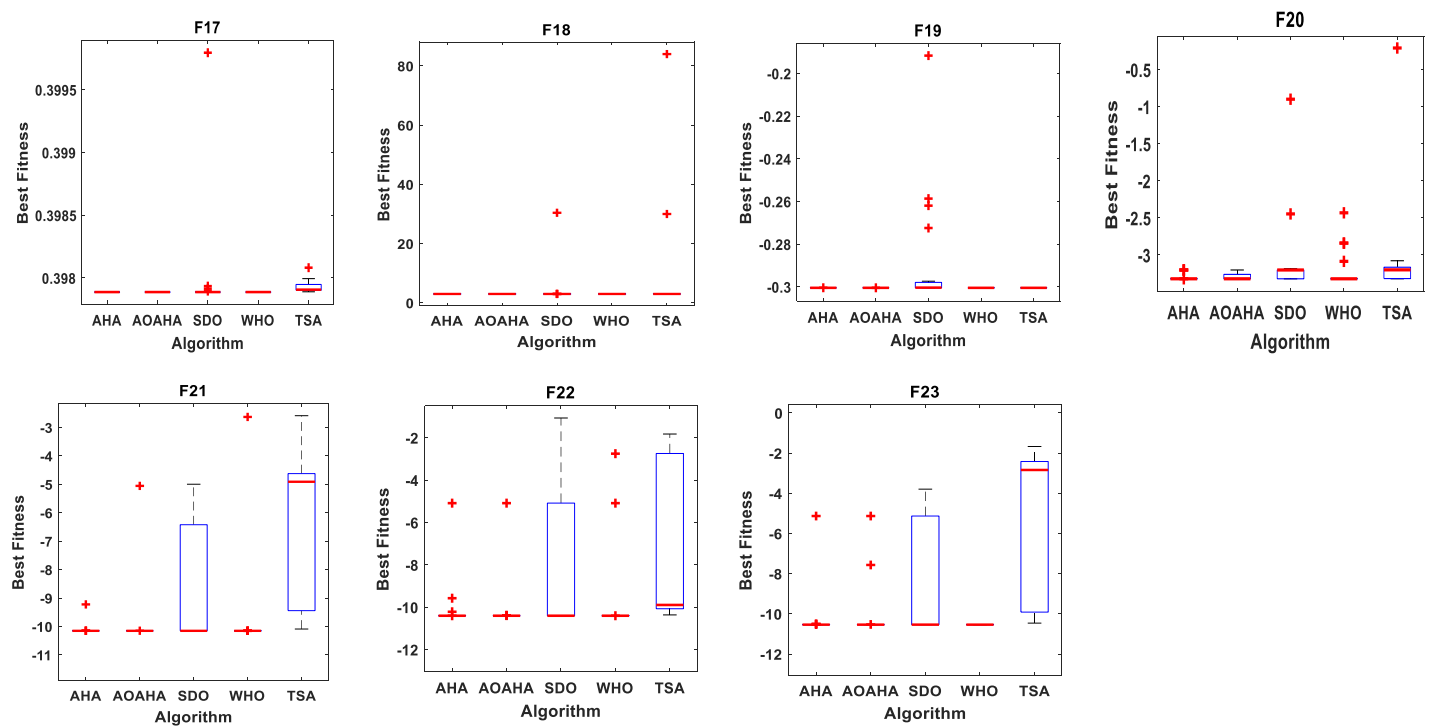

Figure 7. Boxplots for all algorithms for 23 benchmark functions. 


\subsection{Real-World Application}

In this subsection, the AOAHA is evaluated through the application of estimated PV parameters of the static TDM and dynamic IOM/FOM. The obtained results are evaluated and compared with the original algorithm and other recent optimization algorithms.

\subsubsection{Application 1}

In this application, the AOAHA was applied for parameter estimation of the static TDM, using the real data from $57 \mathrm{~mm}$ diameter commercial France R.T.C silicon solar cells. The data were captured under $1000 \mathrm{~W} / \mathrm{m}^{2}$ irradiance, and at a temperature of $33{ }^{\circ} \mathrm{C}$ [10]. Table 4 presents the upper and lower ranges of the nine parameters for the TDM. Table 5 presents the obtained parameters through the proposed and the original algorithms (AOAHA and AHA, respectively) over two more recent algorithms: WOA and BWOA. Table 5 also presents the obtained root-mean-square error (RMSE) (Equation (15)) calculated for all algorithms. It is clear from Table 5 that the AOAHA has the best RMSE value. To present and compare the algorithms' behavior through searching processes, the convergence curves for all compared algorithms are presented in Figure 8. The stability of the results for all compared algorithms was compared through the statistical analysis of the obtained RMSE values from 30 independent runs, and this analysis is presented in Table 6 . The statistical analysis aimed to compare the minimum, maximum, average, and standard deviation values. A graphical presentation of the statistical analysis is presented through the boxplots in Figure 9. An inner-boxplot figure was added to clarify the comparison between the proposed and the original algorithms. The AOAHA achieved the best statistical results for minimum, maximum, average, and standard deviation in terms of stability and robustness. The best fit model to the real data is the model that achieved the least RMSE in comparison to other models, as made clear from the current-voltage characteristics and power-voltage characteristics (Figures 10 and 11, respectively), along with the current absolute error (Equation (16)) and power absolute error (Equation (15)) (Figures 12 and 13, respectively).

$$
\begin{gathered}
\text { RMSE }=\sqrt{\frac{1}{N} \sum_{K=1}^{N} f^{2}\left(V_{t m}, I_{t m}, X\right)} \\
\text { Current Absolute error }=\sqrt[2]{\left(I-I_{\text {estimated }}\right)^{2}} \\
\text { Power Absolute error }=\sqrt[2]{\left(P-P_{\text {estimated }}\right)^{2}}
\end{gathered}
$$

where $f$ is the objective function in Equation (3), $V_{t m}$ and $I_{t m}$ measure the output voltage and current from the real system, respectively, $N$ is the number of measured data, $I$ and $I_{\text {estimated }}$ are the real and estimated output current, respectively, and $P$ and $P_{\text {estimated }}$ are the real and estimated output power, respectively.

Table 4. Upper and lower constraints for all estimated parameters (Application 1).

\begin{tabular}{lll}
\hline Parameter & Solar Cell & \\
\hline & Lower Limit & Upper Limit \\
\hline $\mathrm{R}_{\mathrm{s}}$ & 0 & 5 \\
$\mathrm{R}_{\mathrm{sh}}$ & 0 & 100 \\
$\mathrm{I}_{\mathrm{ph}}$ & 0 & 2 \\
$\mathrm{I}_{\mathrm{s}}$ & 0 & 1 \\
$\mathrm{I}_{\mathrm{s}}$ & 0 & 1 \\
$\mathrm{I}_{\mathrm{s}}$ & 0 & 1 \\
$\eta_{1}$ & 1 & 2 \\
$\eta_{2}$ & 1 & 2 \\
$\eta_{3}$ & 1 & 2 \\
\hline
\end{tabular}


Table 5. Estimated parameters and RMSE of ESGBO and other algorithms for the TDM model.

\begin{tabular}{ccccc}
\hline & AOAHA & AHA & BWOA & WOA \\
\hline $\mathrm{R}_{\mathrm{s}}(\Omega)$ & 0.03674 & 0.036509 & 0.036424 & 0.045276 \\
\hline $\mathrm{R}_{\mathrm{sh}}(\Omega)$ & 55.41315 & 54.16416 & 46.36289 & 15.77225 \\
\hline $\mathrm{I}_{\mathrm{ph}}(\mathrm{A})$ & 0.76078 & 0.760772 & 0.761422 & 0.766883 \\
\hline $\mathrm{I}_{\mathrm{s} 1}(\mathrm{~A})$ & $7.37 \times 10^{-7}$ & $4.00 \times 10^{-8}$ & $1.50 \times 10^{-7}$ & $1.72 \times 10^{-8}$ \\
\hline $\mathrm{I}_{\mathrm{s} 2}(\mathrm{~A})$ & $1.13 \times 10^{-7}$ & $2.98 \times 10^{-7}$ & $1.49 \times 10^{-7}$ & $1.30 \times 10^{-10}$ \\
\hline $\mathrm{I}_{\mathrm{s} 3}(\mathrm{~A})$ & $1.17 \times 10^{-7}$ & $2.39 \times 10^{-8}$ & $1.50 \times 10^{-7}$ & $6.51 \times 10^{-10}$ \\
\hline$\eta_{1}$ & 1.999589 & 1.39016 & 1.504014 & 1.232062 \\
\hline$\eta_{2}$ & 1.46187 & 1.51173 & 1.446121 & 1.85067 \\
\hline$\eta_{3}$ & 1.43712 & 1.562159 & 1.982461 & 1.850237 \\
\hline $\mathrm{RMSE}$ & 0.0009825181 & 0.0009865625 & 0.0010846 & 0.0062131083 \\
\hline
\end{tabular}

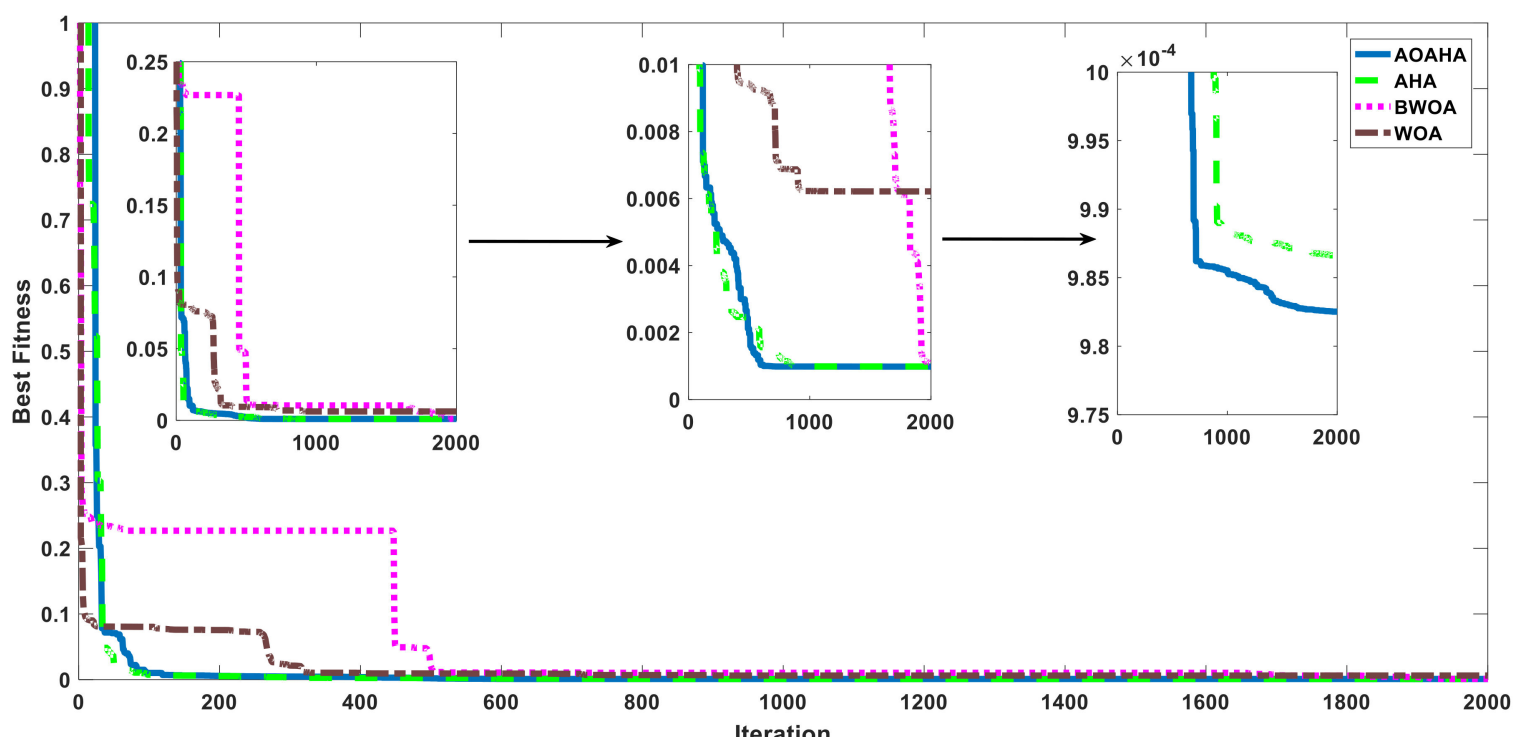

Figure 8. The convergence curve of all algorithms of the TDM.

Table 6. The statistical results of the TDM for all other algorithms.

\begin{tabular}{ccccc}
\hline & Minimum & Average & Maximum & STD \\
\hline AOAHA & 0.0009825181 & 0.000982709 & 0.000982992 & $2.49687 \times 10^{-7}$ \\
\hline AHA & 0.0009865625 & 0.000990229 & 0.000996563 & $5.50757 \times 10^{-6}$ \\
\hline BWOA & 0.0010846 & 0.0017644 & 0.002124 & 0.000589054 \\
\hline WOA & 0.0062131083 & 0.006712806 & 0.007044 & 0.00044033 \\
\hline
\end{tabular}




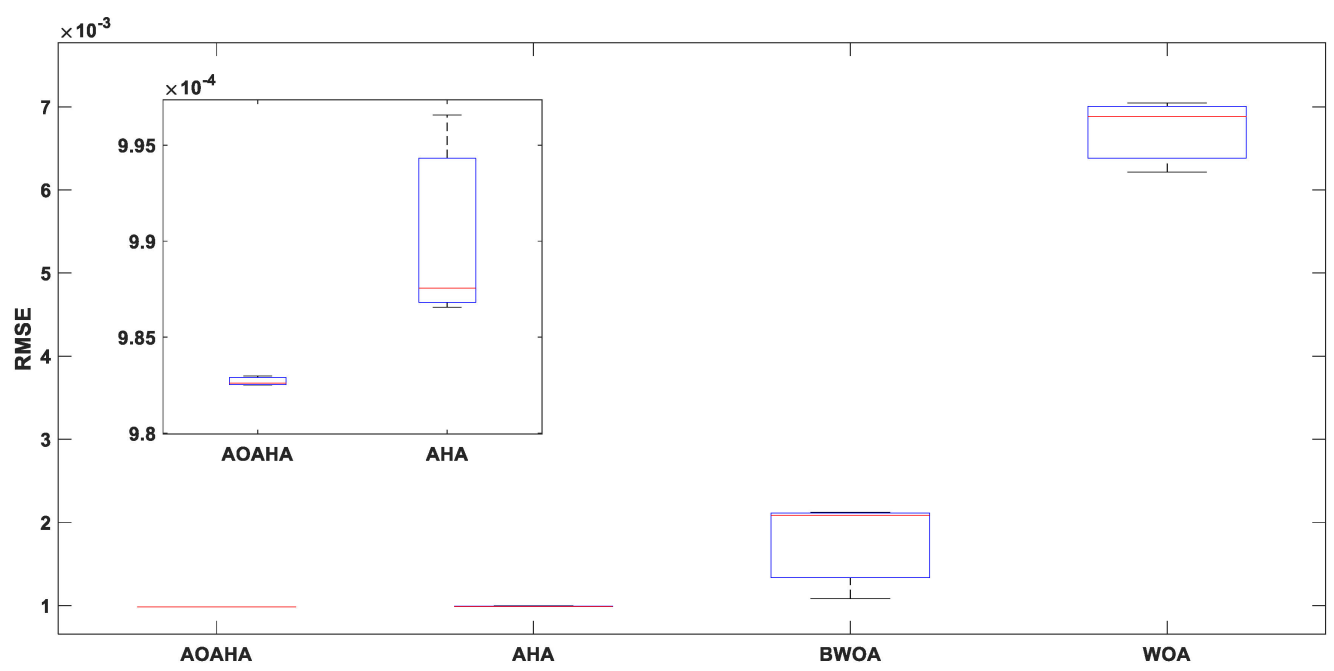

Figure 9. Boxplot figure of all algorithms for 30 independent runs in the case of the TDM.

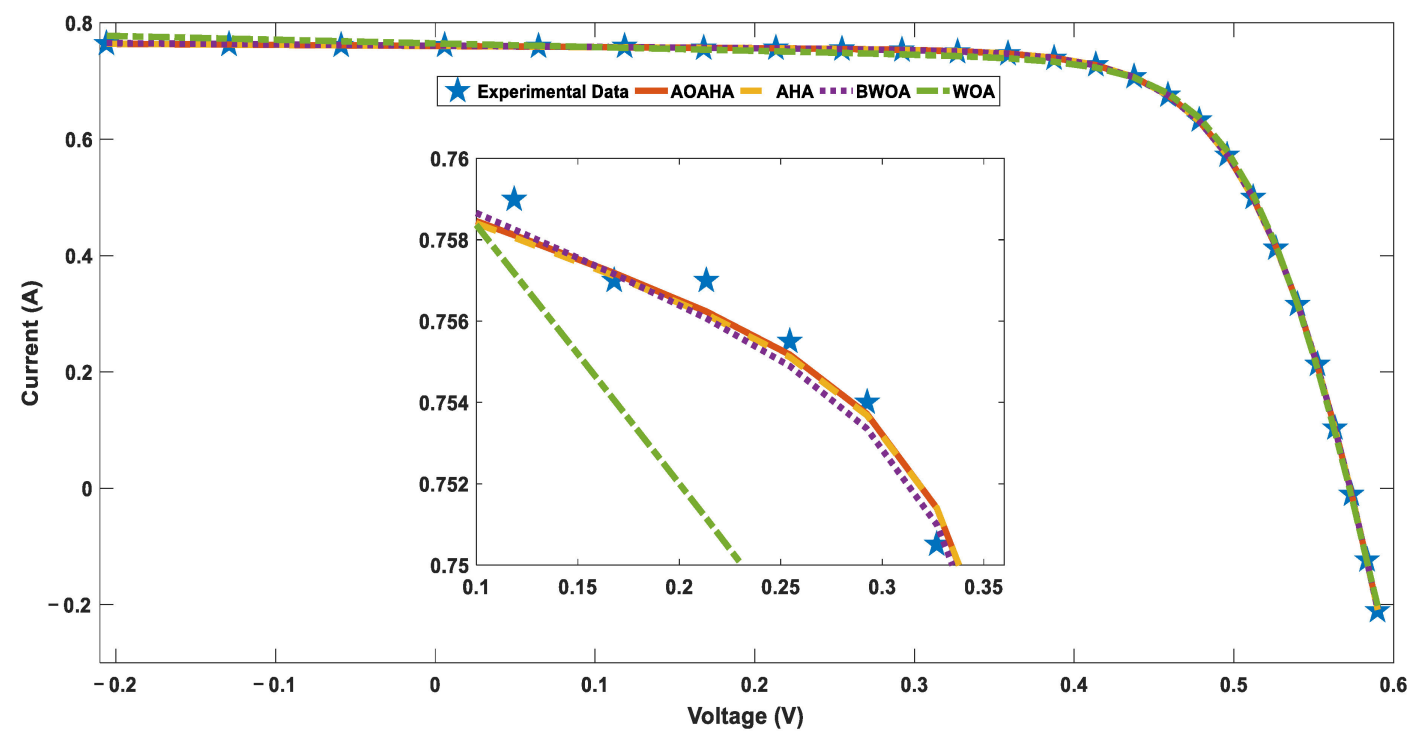

Figure 10. Current-voltage characteristics estimated by all algorithms for the TDM.

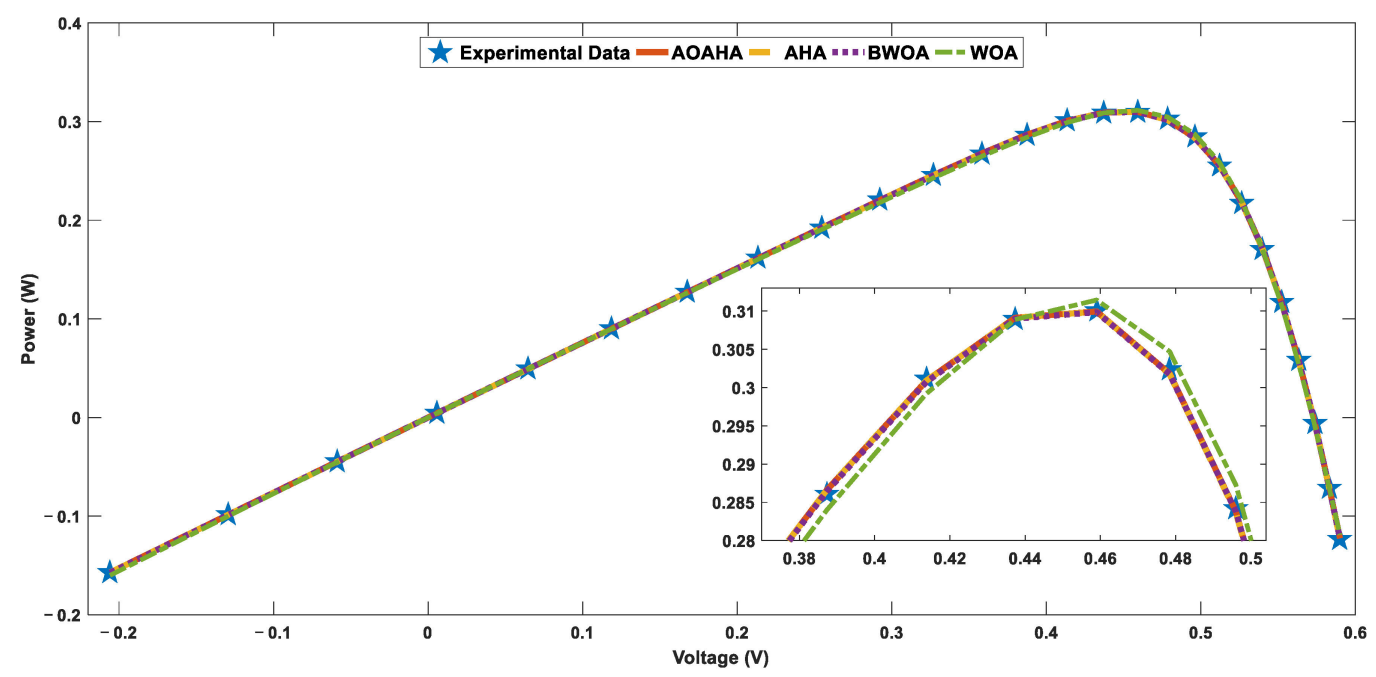

Figure 11. Power-voltage characteristics estimated by all algorithms for the TDM. 


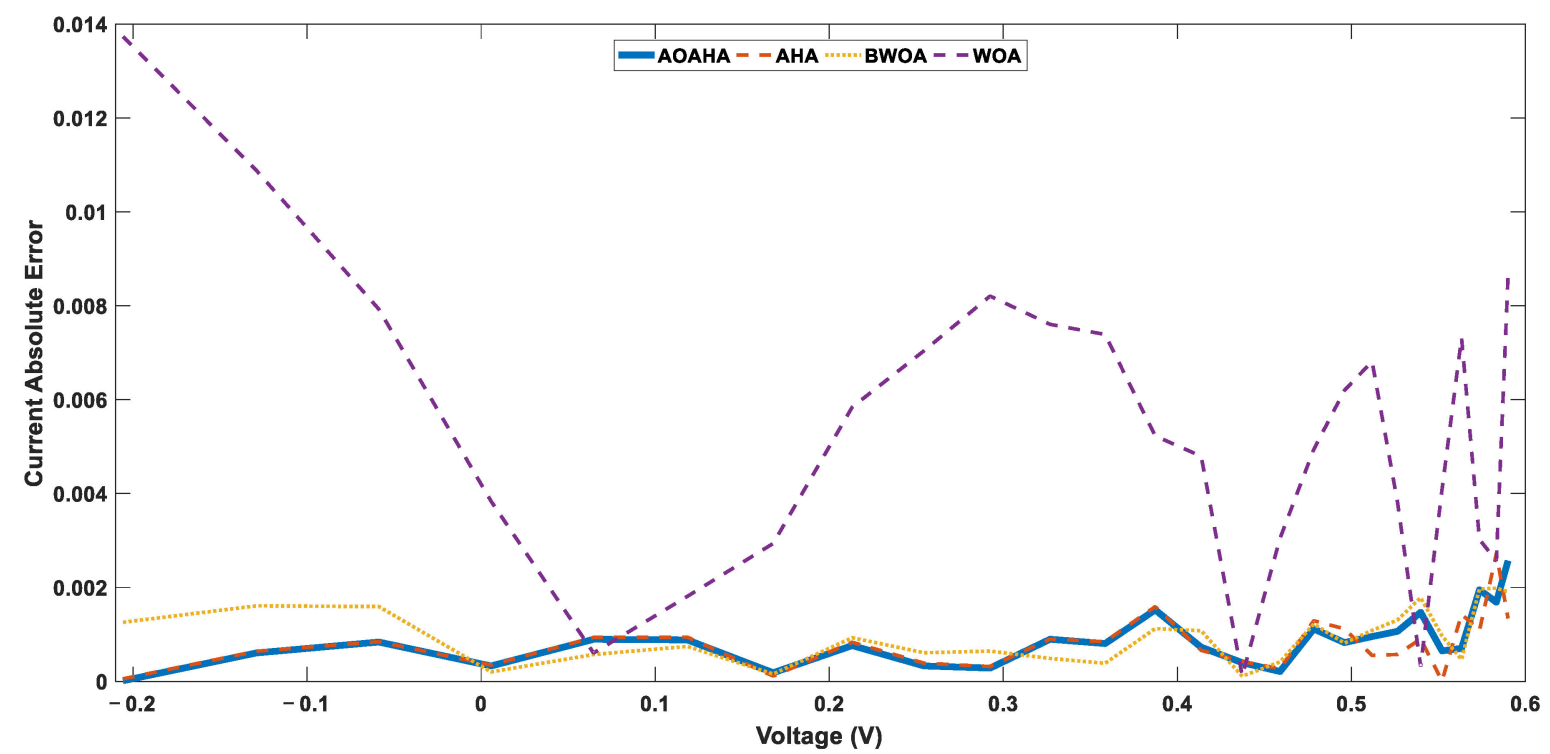

Figure 12. Current absolute error estimated by all algorithms for the TDM.

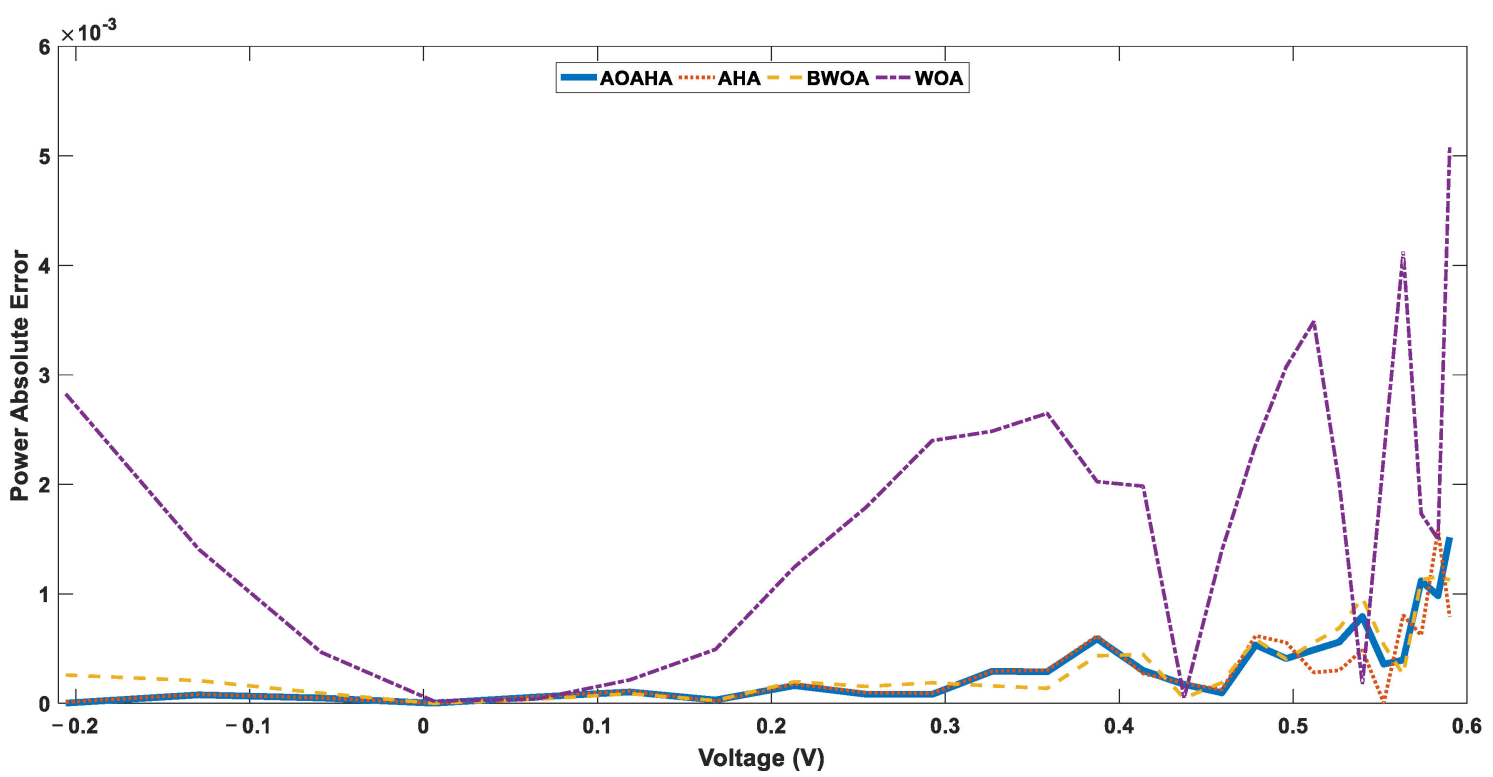

Figure 13. Power absolute error estimated by all algorithms for the TDM.

\subsubsection{Application 2}

In this application, the AOAHA was applied for parameter estimation of the static TDM using the real data from a $7.7 \mathrm{~cm}^{2}$ partition of a multicrystalline Q6-1380 solar cell at different levels of irradiance. The data were captured at room temperature at irradiance of $20 \mathrm{~mW} / \mathrm{m}^{2}, 9.84 \mathrm{~mW} / \mathrm{m}^{2}, 3.47 \mathrm{~mW} / \mathrm{m}^{2}$, and $0.58 \mathrm{~mW} / \mathrm{m}^{2}$ [32]. Table 7 presents the upper and lower ranges of the nine parameters for the TDM. Table 3 presents the obtained parameters through the proposed and the original algorithms (AOAHA and AHA, respectively), along with WOA and BWOA. Table 8 also presents the obtained RMSE calculated for all algorithms at different irradiance. Although the AOAHA has the same RMSE value as the AHA at irradiance od $20 \mathrm{~mW} / \mathrm{cm}^{2}$ and $9.84 \mathrm{~mW} / \mathrm{m}^{2}$, the AOAHA has the best RMSE at irradiance of $3.47 \mathrm{~mW} / \mathrm{m}^{2}$ and $0.58 \mathrm{~mW} / \mathrm{m}^{2}$. Figure 14 presents the RMSE values for all algorithms at different irradiance levels. To present and compare the algorithms' behavior through searching processes, the convergence curves for all compared algorithms are presented in Figure 15. The AOAHA has the best and fastest convergence in 
all cases. The behavior of the TDM model estimated by the AOAHA for different irradiance was compared with real data, as presented in Figure 16.

Table 7. Upper and lower constraints for all estimated parameters (Application 2).

\begin{tabular}{lll}
\hline Parameter & Solar Cell & \\
\hline & Lower Limit & Upper Limit \\
\hline$R_{\mathrm{s}}$ & 0 & 5 \\
$\mathrm{R}_{\mathrm{sh}}$ & 0 & 5000 \\
$\mathrm{I}_{\mathrm{ph}}$ & 0 & 2 \\
$\mathrm{I}_{\mathrm{s} 1}$ & 0 & 1 \\
$\mathrm{I}_{\mathrm{s} 2}$ & 0 & 1 \\
$\mathrm{I}_{\mathrm{s} 3}$ & 0 & 1 \\
$\eta_{1}$ & 1 & 50 \\
$\eta_{2}$ & 1 & 50 \\
$\eta_{3}$ & 1 & 50 \\
\hline
\end{tabular}

Table 8. The estimated parameters and RMSE for algorithms at different irradiance.

\begin{tabular}{|c|c|c|c|c|c|c|c|c|c|c|c|}
\hline \multirow{2}{*}{$\begin{array}{l}\text { Irradiance } \\
\text { Level }\end{array}$} & & \multicolumn{10}{|c|}{ Parameters } \\
\hline & & $\mathbf{R}_{\mathrm{s}}$ & $\mathbf{R}_{\mathrm{sh}}$ & $\mathbf{I}_{\mathrm{ph}}$ & $I_{\mathrm{s} 1}$ & $I_{\mathrm{s} 2}$ & $\mathrm{I}_{\mathrm{s} 3}$ & $\eta_{1}$ & $\eta_{2}$ & $\eta_{3}$ & RMSE \\
\hline \multirow{3}{*}{$\begin{array}{c}\text { At } 20 \\
\mathrm{mw} / \mathrm{cm}^{2}\end{array}$} & AOAHA & 0.0895 & 5000.00 & 0.0399 & 0.0005289 & $\begin{array}{l}2.08 \times \\
10^{-10}\end{array}$ & $\begin{array}{l}2.39 \times \\
10^{-10}\end{array}$ & 4.1698 & 4.1698 & 4.1698 & $2.23 \times 10^{-4}$ \\
\hline & AHA & 0.0895 & 5000.00 & 0.0399 & $\begin{array}{c}2.33 \times \\
10^{-10}\end{array}$ & 0.000528 & $\begin{array}{l}4.37 \times \\
10^{-10}\end{array}$ & 4.1699 & 4.1698 & 4.1699 & $2.23 \times 10^{-4}$ \\
\hline & BWOA & 0.0914 & 5000.00 & 0.0399 & 0.0005271 & $\begin{array}{c}1.16 \times \\
10^{-6}\end{array}$ & $\begin{array}{c}6.08 \times \\
10^{-16}\end{array}$ & 4.1664 & 27.1378 & 27.1375 & $2.23 \times 10^{-4}$ \\
\hline \multirow{5}{*}{$\begin{array}{c}\text { At } 9.84 \\
\mathrm{mw} / \mathrm{cm}^{2}\end{array}$} & WOA & 0.3963 & 208.77 & 0.0399 & $\begin{array}{l}1.18 \times \\
10^{-20}\end{array}$ & $\begin{array}{l}1.07 \times \\
10^{-20}\end{array}$ & 0.0002302 & 25.4707 & 1.0696 & 3.5395 & $3.68 \times 10^{-4}$ \\
\hline & AOAHA & 0.7072 & 579.49 & 0.0197 & 0.000247 & $\begin{array}{c}2.95 \times \\
10^{-5}\end{array}$ & 0.0001220 & 3.4987 & 49.2649 & 46.6918 & $1.32 \times 10^{-4}$ \\
\hline & AHA & 0.7057 & 545.48 & 0.0197 & $\begin{array}{c}1.49 \times \\
10^{-10}\end{array}$ & $\begin{array}{c}9.74 \times \\
10^{-10}\end{array}$ & 0.00024880 & 38.9430 & 45.8783 & 3.5019 & $1.32 \times 10^{-4}$ \\
\hline & BWOA & 0.7057 & 545.52 & 0.0197 & 0.0002488 & $\begin{array}{l}1.00 \times \\
10^{-20}\end{array}$ & $\begin{array}{l}1.00 \times \\
10^{-20}\end{array}$ & 3.5019 & 4.8614 & 29.4960 & $1.32 \times 10^{-4}$ \\
\hline & BWOA & 0.1759 & 591.45 & 0.0197 & 0.0004273 & $\begin{array}{l}2.02 \times \\
10^{-20}\end{array}$ & $\begin{array}{l}2.02 \times \\
10^{-20}\end{array}$ & 3.9971 & 1.9377 & 2.0179 & $2.20 \times 10^{-4}$ \\
\hline \multirow{3}{*}{$\begin{array}{l}\text { At } 3.47 \\
\mathrm{mw} / \mathrm{cm}^{2}\end{array}$} & AOAHA & 1.1300 & 611.16 & 0.0070 & $\begin{array}{c}2.64 \times \\
10^{-8}\end{array}$ & $\begin{array}{l}1.76 \times \\
10^{-4}\end{array}$ & $\begin{array}{c}1.30 \times \\
10^{-9}\end{array}$ & 40.9859 & 3.1423 & 39.6484 & $8.26 \times 10^{-5}$ \\
\hline & AHA & 1.1330 & 801.83 & 0.0070 & $\begin{array}{c}3.57 \times \\
10^{-6}\end{array}$ & $\begin{array}{c}1.73 \times \\
10^{-4}\end{array}$ & 0.00052421 & 44.3793 & 3.1341 & 47.8092 & $8.27 \times 10^{-5}$ \\
\hline & BWOA & 1.1301 & 611.05 & 0.0070 & $\begin{array}{l}1.41 \times \\
10^{-19}\end{array}$ & $\begin{array}{l}1.86 \times \\
10^{-15}\end{array}$ & 0.00017578 & 49.9954 & 9.5640 & 3.1422 & $8.26 \times 10^{-5}$ \\
\hline \multirow{5}{*}{$\begin{array}{c}\text { At } 0.58 \\
\mathrm{mw} / \mathrm{cm}^{2}\end{array}$} & BWOA & 0.0752 & 1224.95 & 0.0070 & $\begin{array}{c}7.02 \times \\
10^{-20}\end{array}$ & 0.000370 & $\begin{array}{l}2.35 \times \\
10^{-20}\end{array}$ & 6.0038 & 3.8487 & 45.0651 & $1.49 \times 10^{-4}$ \\
\hline & AOAHA & 1.4704 & 4996.03 & 0.0014 & 0.000748 & 0.000105 & $\begin{array}{c}3.25 \times \\
10^{-6}\end{array}$ & 10.2939 & 2.8197 & 14.9268 & $7.15 \times 10^{-5}$ \\
\hline & AHA & 1.5406 & 4944.53 & 0.0014 & $\begin{array}{c}7.91 \times \\
10^{-5}\end{array}$ & $\begin{array}{c}4.14 \times \\
10^{-6}\end{array}$ & 0.00065080 & 2.6899 & 21.0457 & 7.9911 & $7.10 \times 10^{-5}$ \\
\hline & BWOA & 1.2021 & 956.54 & 0.0014 & 0.0005149 & 0.000173 & $\begin{array}{l}1.00 \times \\
10^{-20}\end{array}$ & 17.6110 & 3.1138 & 49.9999 & $7.37 \times 10^{-5}$ \\
\hline & BWOA & 0.4513 & 1133.55 & 0.0014 & 0.0003144 & $\begin{array}{l}1.60 \times \\
10^{-19}\end{array}$ & $\begin{array}{l}5.35 \times \\
10^{-19}\end{array}$ & 3.6546 & 43.9120 & 45.4858 & $8.48 \times 10^{-5}$ \\
\hline
\end{tabular}




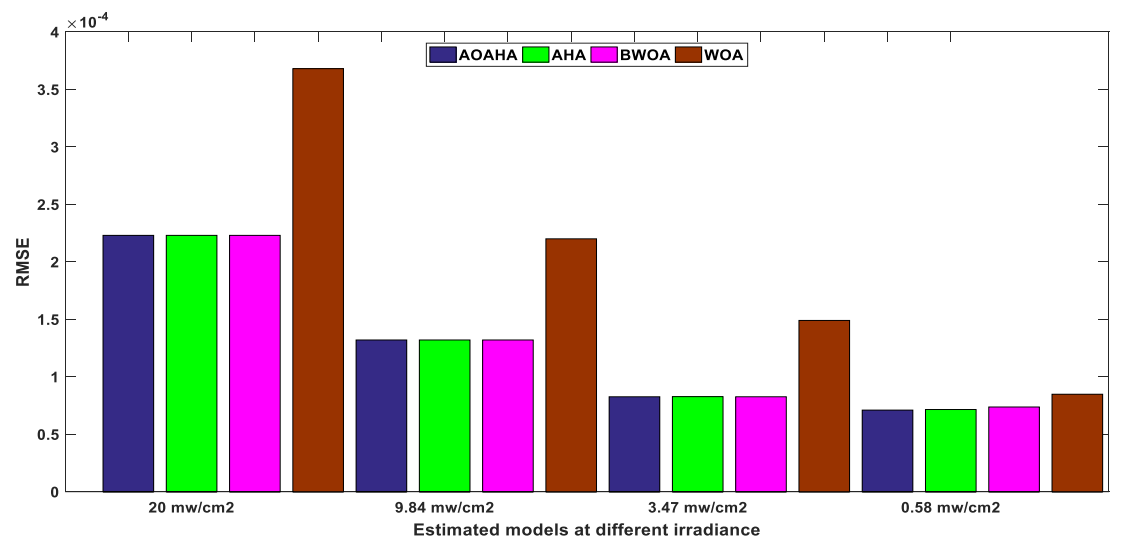

Figure 14. Summary of the obtained RMSE by all algorithms at different irradiances.
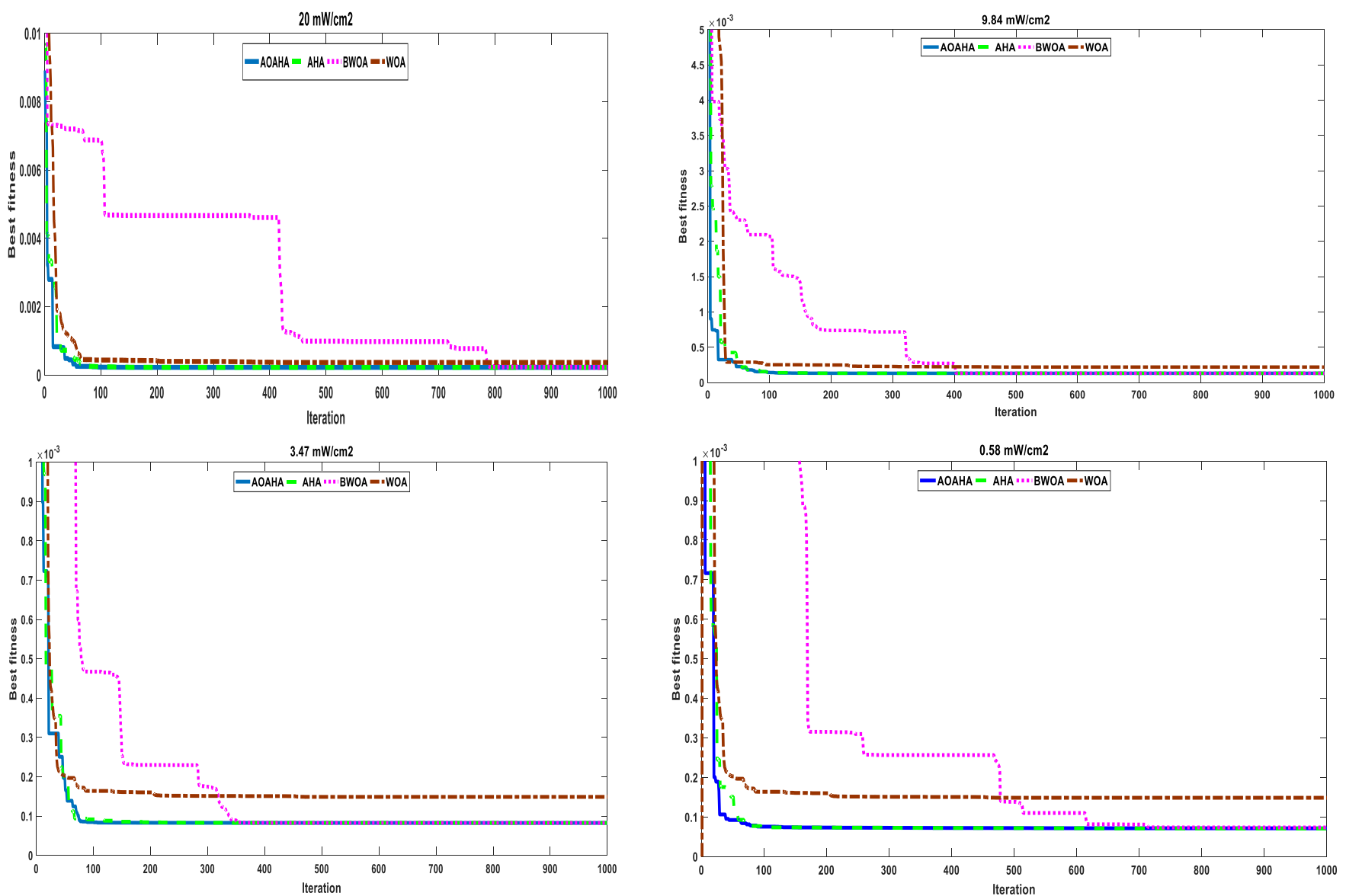

Figure 15. Convergence curves for all algorithms at different irradiances. 


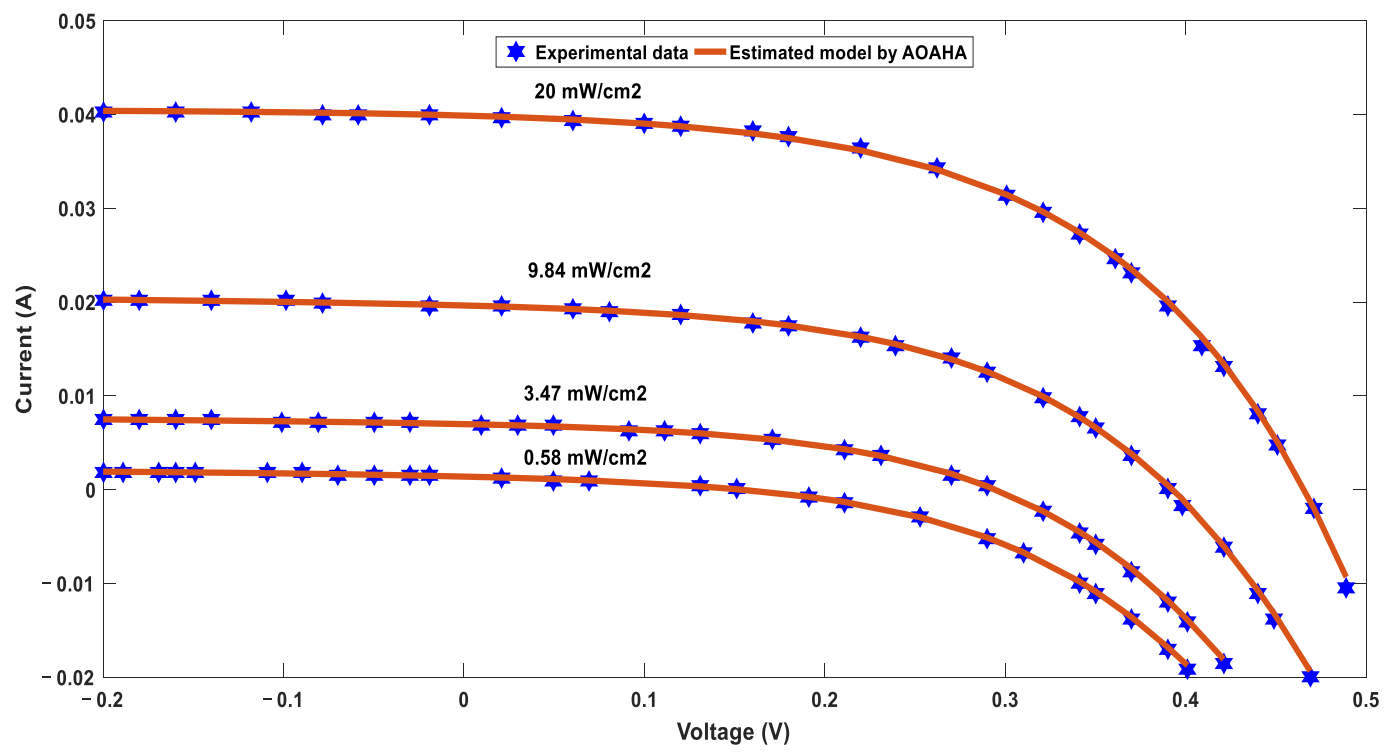

Figure 16. Voltage-current characteristic curves for the real data and AOAHA at different irradiances.

\subsubsection{Application 3}

In this application, the AOAHA was applied for parameter estimation of dynamic PV models (IOM and FOM) using the real data from a PV module at a temperature of $25^{\circ} \mathrm{C}$ and irradiance of $655 \mathrm{~W} / \mathrm{m}^{2}$.The connected load $\left(\mathrm{R}_{1}\right)$ was $23.1 \mathrm{ohm}$. Table 9 presents the upper and lower ranges of the three parameters for the IOM and the five parameters for the FOM.

Table 9. Upper and lower constraints for all estimated parameters (Application 3).

\begin{tabular}{|c|c|c|}
\hline \multirow[t]{2}{*}{ Parameter } & \multicolumn{2}{|l|}{ Solar Cell } \\
\hline & Lower Limit & Upper Limit \\
\hline $\mathrm{R}_{\mathrm{c}}$ & 0 & 20 \\
\hline C & $2 \times 10^{-8}$ & $6 \times 10^{-5}$ \\
\hline $\mathrm{L}$ & $5 \times 10^{-6}$ & $1 \times 10^{-4}$ \\
\hline$\alpha$ & 0.8 & 1.1 \\
\hline$\beta$ & 0.8 & 1.1 \\
\hline
\end{tabular}

Tables 10 and 11 present the obtained parameters from the proposed and the original algorithm for the IOM and FOM, respectively; they also present the RMSE obtained for all compared algorithms for the IOM and FOM, respectively. From the RMSE values, the AOAHA and AHA have the same results, which are better than those of the other algorithms; additionally, the results obtained by the FOM are more accurate than those obtained by the IOM model. To present and compare the algorithms' behavior through the searching processes of the IOM and FOM, the convergence curves for all compared algorithms are presented in Figures 17 and 18, respectively. The statistical analysis of 30 independent runs for the IOM and FOM are presented in Tables 12 and 13, respectively, and graphically presented in boxplots in Figures 19 and 20, respectively. From the IOM statistical analysis, although the AOAHA and AHA have the same minimum values, the AOAHA has better average, maximum, and standard deviation values than the AHA and all other compared algorithms. From the FOM statistical analysis, although the AHA has better standard deviation than the AOAHA, the latter has better minimum, average, and maximum values than the AHA and all other compared algorithms. Figures 21 and 22 present the load-current curve for the real experimental data and all algorithms for the IOM and FOM, respectively. 
Table 10. Estimated parameters of the IOM model for all algorithms.

\begin{tabular}{ccccc}
\hline & AOAHA & AHA & WOA & BWOA \\
\hline $\mathrm{R}_{\mathrm{c}}$ & 13.79387624 & 13.79388 & 13.06099 & 13.06404 \\
\hline $\mathrm{C}$ & $1.57 \times 10^{-6}$ & $1.57 \times 10^{-6}$ & $1.70 \times 10^{-6}$ & $1.71 \times 10^{-6}$ \\
\hline $\mathrm{L}$ & $7.50 \times 10^{-6}$ & $7.50 \times 10^{-6}$ & $7.50 \times 10^{-6}$ & $7.50 \times 10^{-6}$ \\
\hline $\mathrm{RMSE}$ & 0.008403 & 0.008403 & 0.008409 & 0.008409 \\
\hline
\end{tabular}

Table 11. Estimated parameters of the FOM model for all algorithms.

\begin{tabular}{ccccc}
\hline & AOAHA & AHA & WOA & BWOA \\
\hline $\mathrm{R}_{\mathrm{c}}$ & 6.668716 & 6.552183 & 3.513184 & 6.162661 \\
\hline $\mathrm{C}$ & $9.92 \times 10^{-6}$ & $9.31 \times 10^{-6}$ & $1.47 \times 10^{-5}$ & $3.23 \times 10^{-6}$ \\
\hline $\mathrm{L}$ & $1.65 \times 10^{-5}$ & $1.69 \times 10^{-5}$ & $9.48 \times 10^{-5}$ & $2.12 \times 10^{-5}$ \\
\hline$\alpha$ & 0.845127 & 0.84893 & 0.807758 & 0.93991 \\
\hline$\beta$ & 0.945356 & 0.943134 & 0.823822 & 0.927025 \\
\hline RMSE & 0.007712 & 0.007712 & 0.009177 & 0.008011 \\
\hline
\end{tabular}

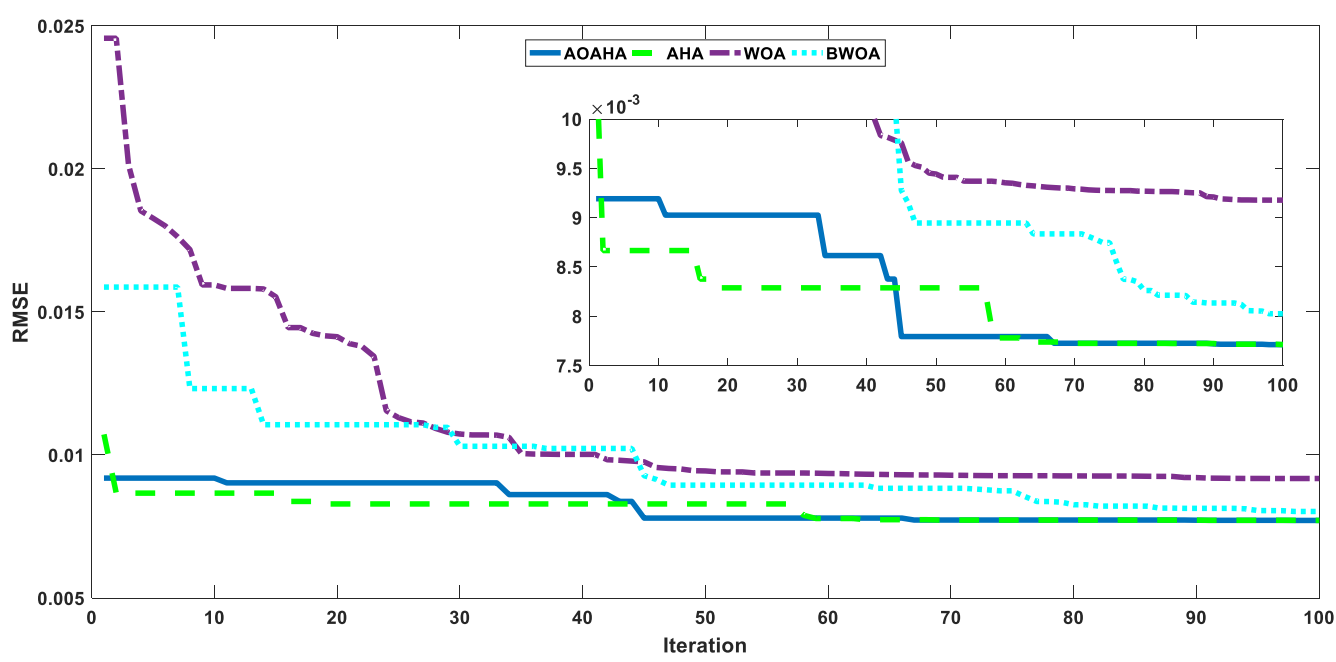

Figure 17. Convergence curves of all algorithms for the IOM. 


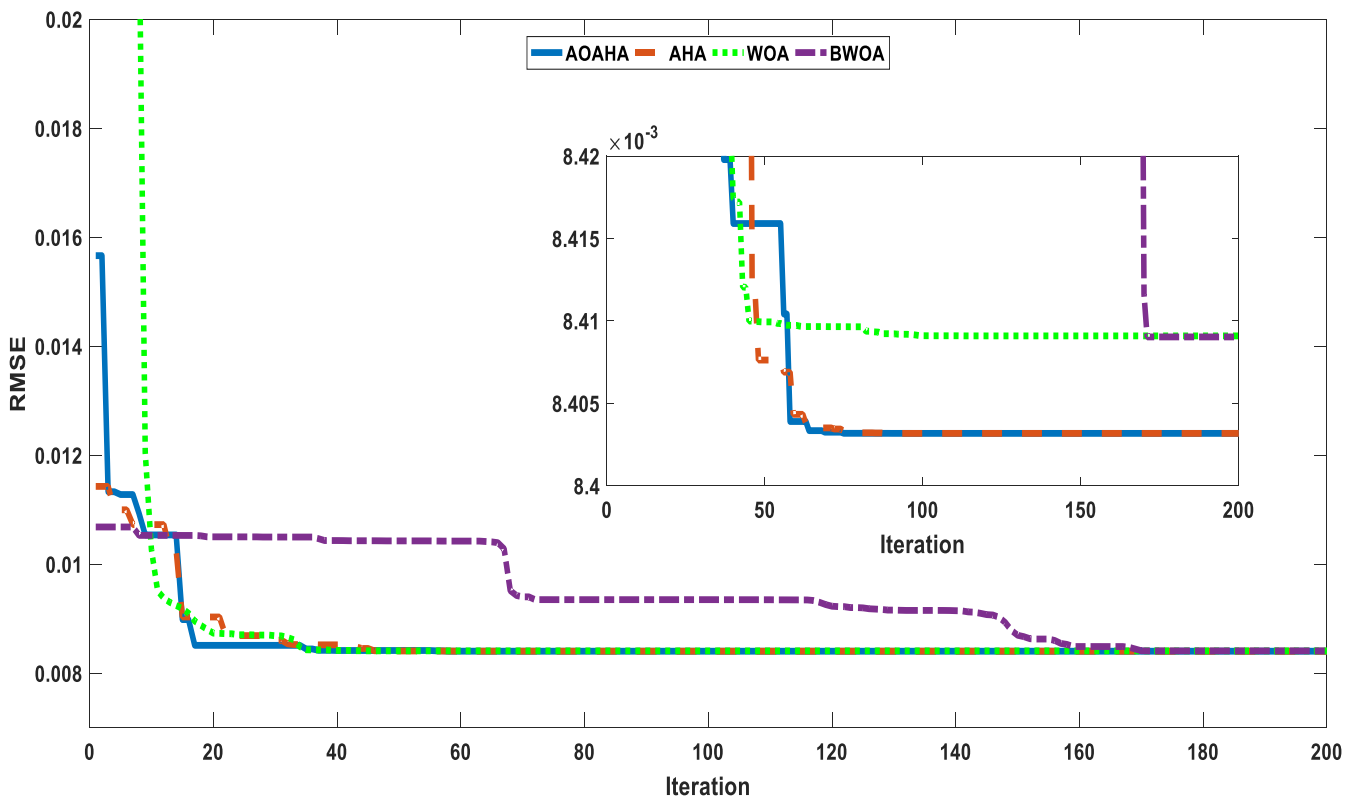

Figure 18. Convergence curves of all algorithms for the FOM.

Table 12. The statistical results of the IOM for all other algorithms.

\begin{tabular}{ccccc}
\hline & Minimum & Average & Maximum & STD \\
\hline AOAHA & 0.008403003 & 0.008403012 & 0.008403032 & $1.16558 \times 10^{-8}$ \\
\hline AHA & 0.008403003 & 0.008403046 & 0.008403207 & $9.03883 \times 10^{-8}$ \\
\hline WOA & 0.008409 & 0.008409 & 0.00841 & $3.94 \times 10^{-7}$ \\
\hline BWOA & 0.008409 & 0.008409 & 0.00841 & $3.36 \times 10^{-7}$ \\
\hline
\end{tabular}

Table 13. The statistical results of the FOM for all other algorithms.

\begin{tabular}{ccccc}
\hline & Minimum & Average & Maximum & STD \\
\hline AOAHA & 0.0076253586 & 0.0077234518 & 0.0078285384 & $9.06109 \times 10^{-5}$ \\
\hline AHA & 0.0077604697 & 0.0078421887 & 0.0079199453 & $6.59704 \times 10^{-5}$ \\
\hline WOA & 0.009177 & 0.0091974 & 0.009278 & $4.50571 \times 10^{-5}$ \\
\hline BWOA & 0.0080114 & 0.0080315 & 0.008111 & $4.44423 \times 10^{-5}$ \\
\hline
\end{tabular}




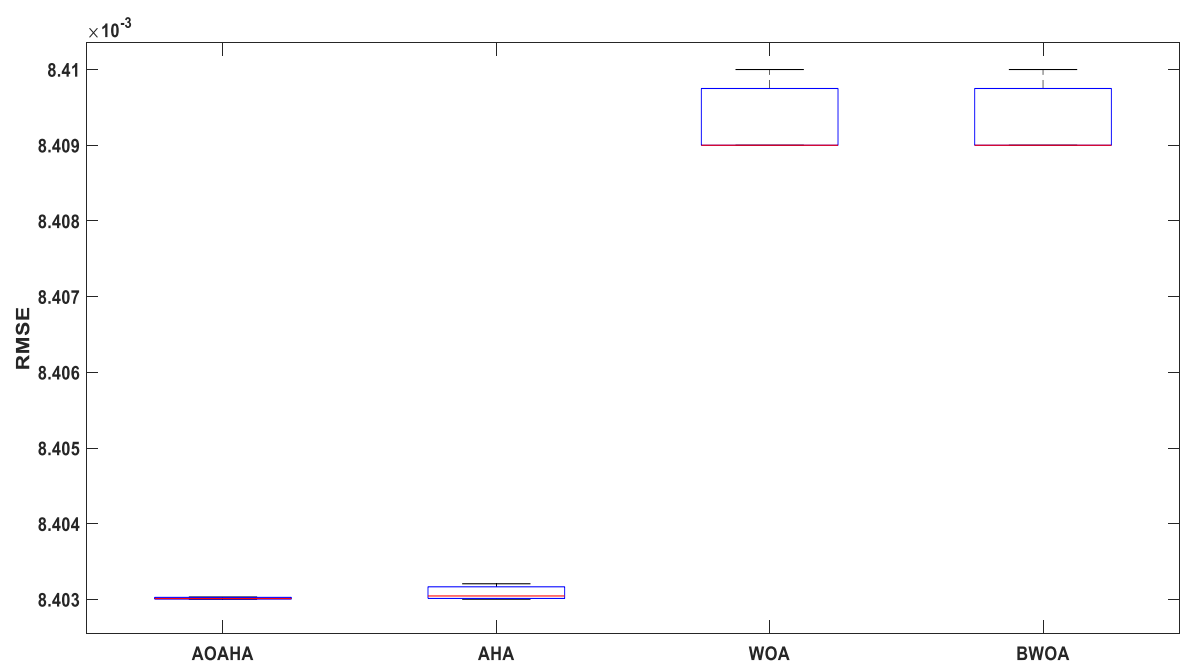

Figure 19. Boxplot figure of all algorithms for 30 independent runs in the case of the IOM.

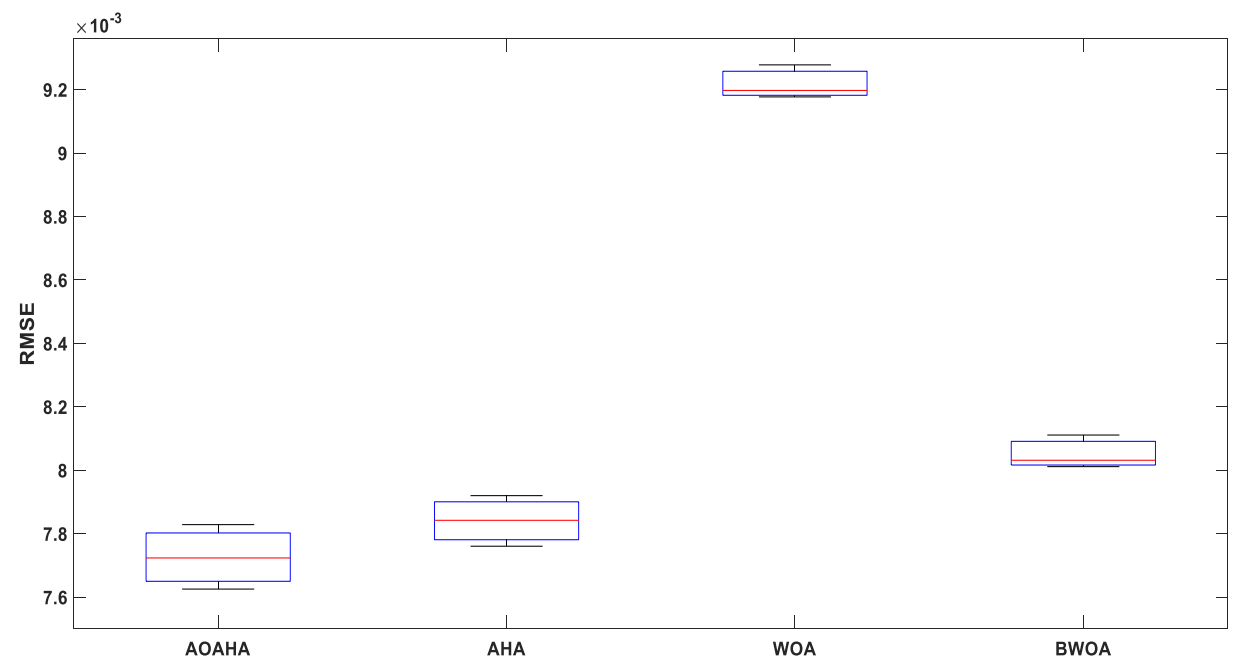

Figure 20. Boxplot figure of all algorithms for 30 independent runs in the case of the FOM.

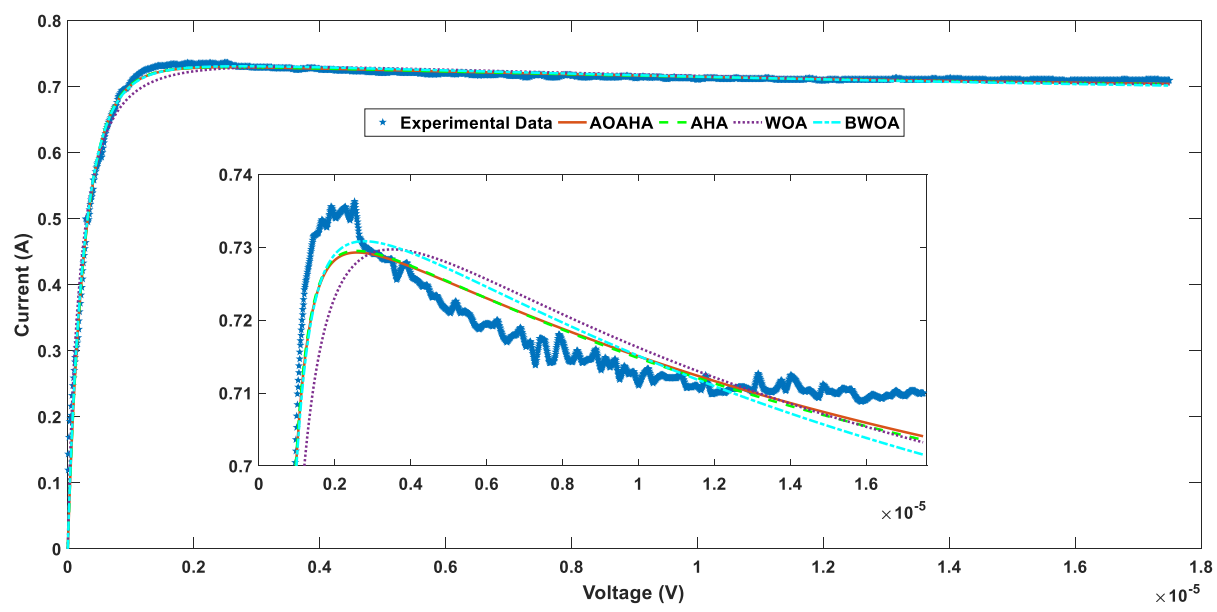

Figure 21. Load-current curve of real data and the estimated IOM by different algorithms. 


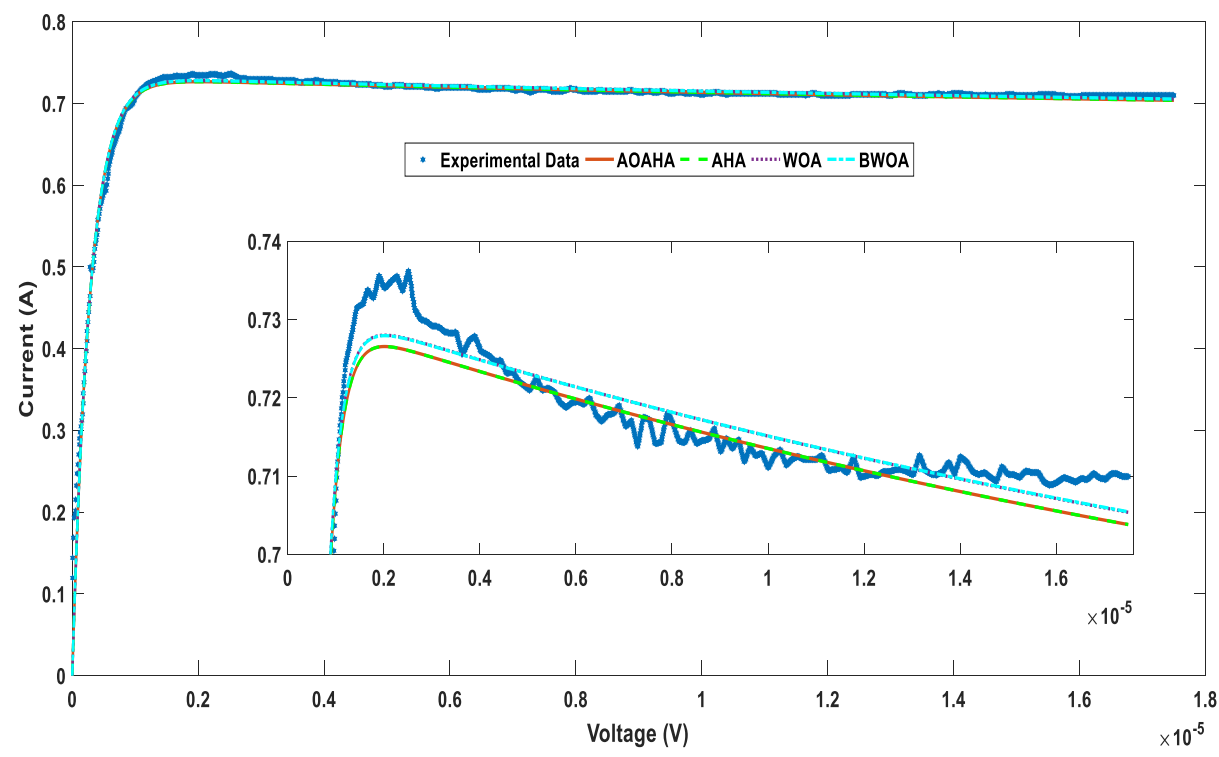

Figure 22. Load-current curve of real data and the estimated FOM by different algorithms.

Figures 23 and 24 present the current absolute error curve of all algorithms for the IOM and FOM, respectively.

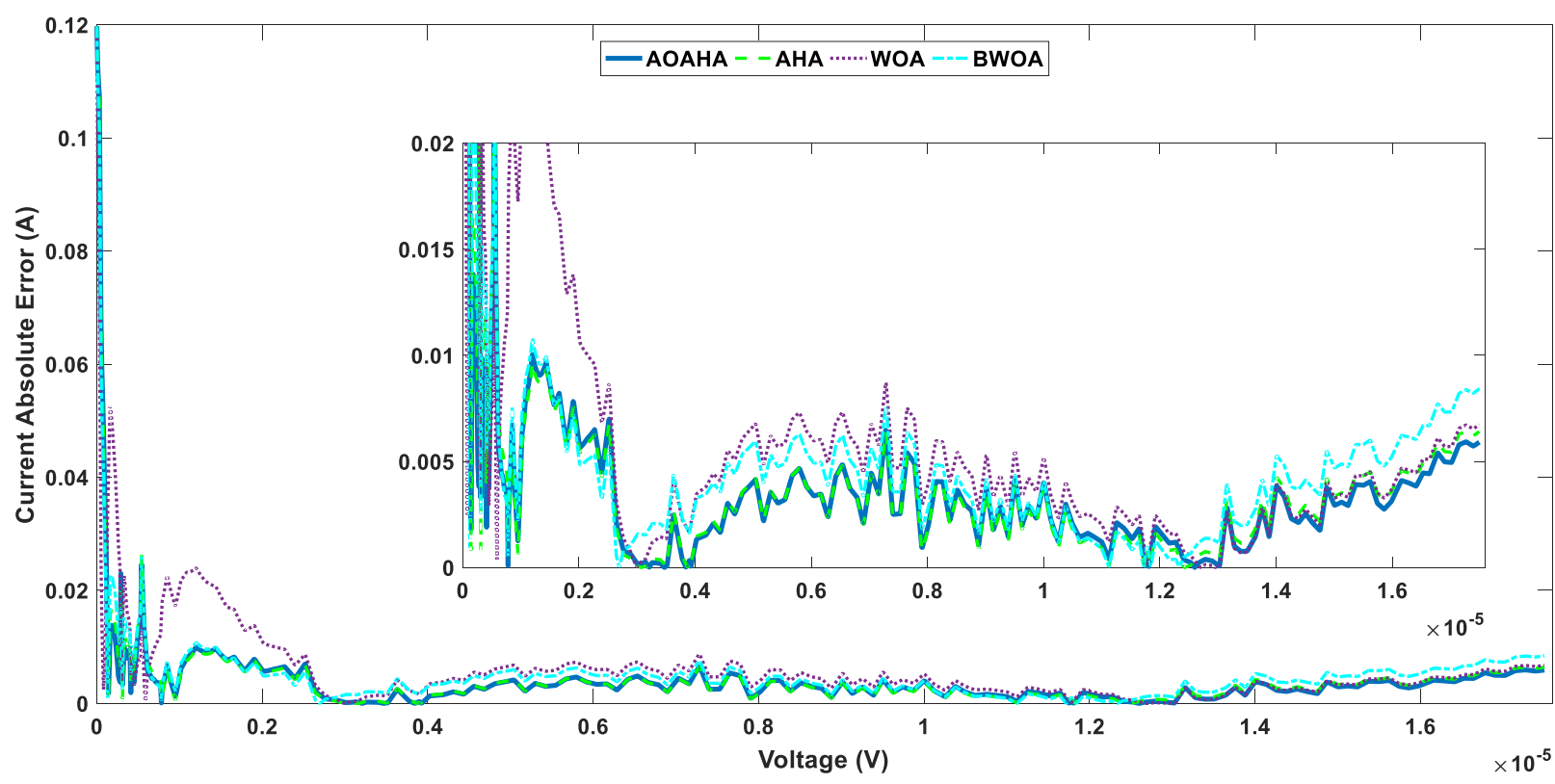

Figure 23. Current absolute error of the estimated IOM by different algorithms. 


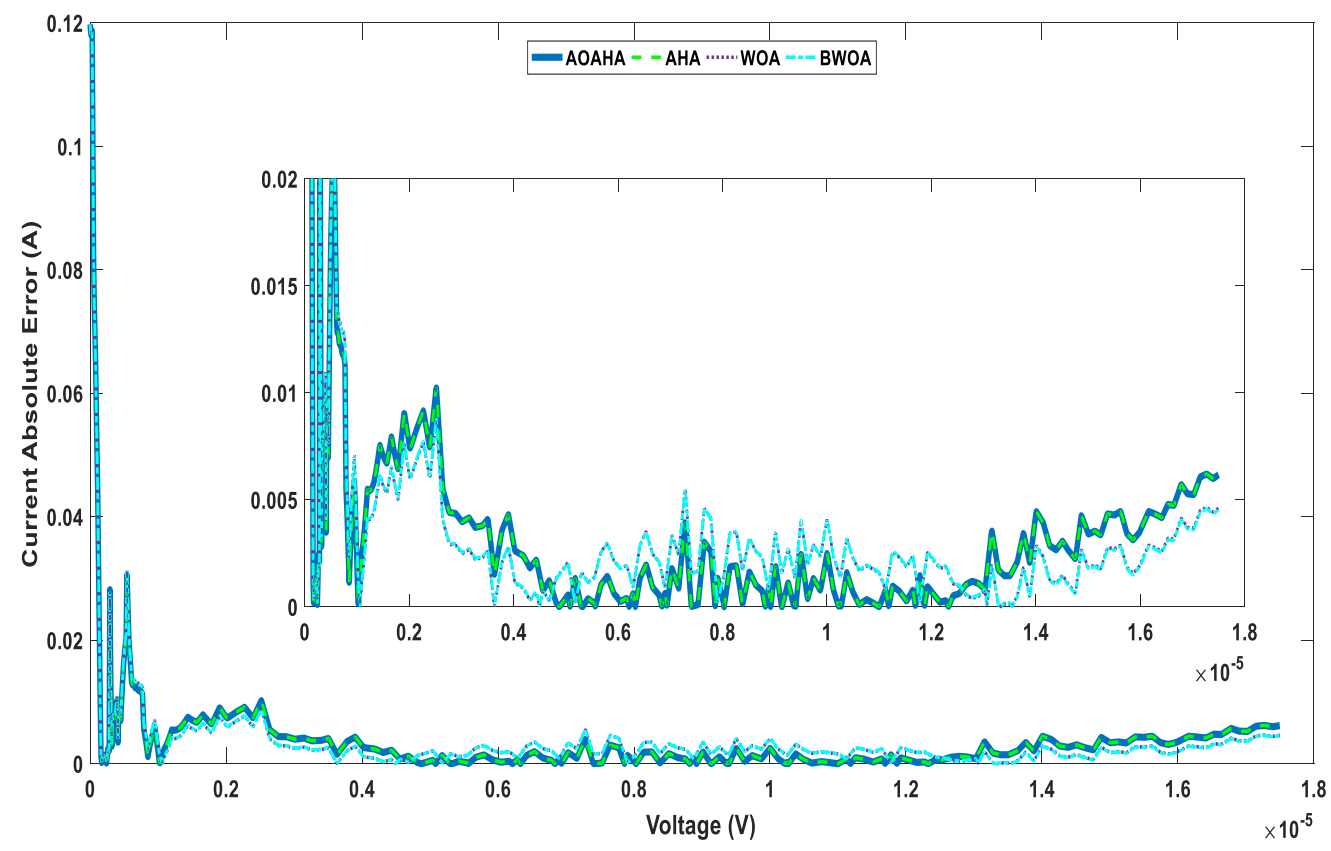

Figure 24. Current absolute error of the estimated FOM by different algorithms.

\section{Conclusions}

In this paper, a novel enhanced optimization algorithm was proposed. The enhanced algorithm is called the AOAHA. The enhancement is based on adding an adaptive part to the original AHA algorithm. The new algorithm was evaluated though benchmark functions and through real applications. The benchmark functions comprised 23 unimodal, multimodal, and composite functions. The real applications were for the parameter estimation of a PV static TDM for $57 \mathrm{~mm}$ diameter commercial France R.T.C silicon solar cells, multicrystalline Q6-1380 solar cells at different levels of irradiance, and dynamic IOM and dynamic FOM for a PV module at a temperature of $25{ }^{\circ} \mathrm{C}$ and irradiance level of $655 \mathrm{~W} / \mathrm{m}^{2}$ through a connected load of $\mathrm{R}_{1}=23.1$. The obtained results for all tests were evaluated by comparing the results using different factors. RMSE and IAE were used to check the accuracy. The algorithm robustness was tested by running the algorithms in 30 independent runs and comparing the obtained results through statistical analysis. The values calculated in the statistical analysis were the minimum, maximum, average, and standard deviation. The AOAHA had the best performance in the majority of the tests. From the RMSE obtained for the IOM and FOM, the dynamic FOM is more accurate than the IOM. Different types of PV models and different applications, along with different operation conditions, were used to test the proposed algorithm from different sides, and satisfied a good performance in all tests. For future work, the proposed algorithm can be applied to estimate PV model parameters for large PV systems and complex problems such as optimal power flow economic emission dispatch with renewable energy resources for large scale power systems and optimal residential load scheduling for photovoltaic systems [33].

Author Contributions: Conceptualization, A.R., S.K. and M.H.H.; data curation, E.M.A. and H.M.H.; formal analysis, A.R., S.K. and M.H.H.; funding acquisition, E.M.A. and H.M.H.; investigation, A.R., S.K. and M.H.H.; methodology, A.R., S.K. and M.H.H.; project administration, E.M.A. and H.M.H.; resources, E.M.A. and H.M.H.; software, A.R., M.H.H. and S.K.; supervision, E.M.A. and H.M.H.; validation, A.R., S.K. and M.H.H.; visualization, E.M.A. and H.M.H.; writing-original draft, A.R., S.K. and M.H.H.; writing - review and editing, E.M.A., and H.M.H. All authors have read and agreed to the published version of the manuscript.

Funding: This work is funded by the Deanship of Scientific Research at Jouf University under grant No (DSR-2021-02-0311). 
Institutional Review Board Statement: Not applicable.

Informed Consent Statement: Not applicable.

Data Availability Statement: Not applicable.

Conflicts of Interest: The authors declare no conflict of interest.

\section{Nomenclature}

\begin{tabular}{|c|c|}
\hline Symbol & Description \\
\hline TDM & Three-diode model \\
\hline DDM & Double-diode model \\
\hline SDM & Single-diode model \\
\hline IOM & Integral order model \\
\hline FOM & Fractional order model \\
\hline AHA & Artificial hummingbird algorithm \\
\hline AOAHA & Adaptive opposition artificial hummingbird algorithm \\
\hline PV & Photovoltaic \\
\hline $\mathrm{V}$ & Terminal voltage \\
\hline I & PV module output current \\
\hline $\mathrm{I}_{\mathrm{ph}}$ & Current source generated from the photons \\
\hline RMSE & Root-mean-square error \\
\hline$\eta_{1}$ & Ideality factor for the first diode (diffusion of current components) \\
\hline$\eta_{2}$ & Ideality factor for the second diode (recombination of current components) \\
\hline $\mathrm{T}(\mathrm{Ko})$ & Photocell temperature (Kelvin) \\
\hline$\eta_{3}$ & Ideality factor for the third diode (leakage of current components) \\
\hline Rs & $\begin{array}{l}\text { Series resistance to represent the total resistance of the } \\
\text { semiconductor material at neutral regions }\end{array}$ \\
\hline $\mathrm{R}_{\mathrm{sh}}$ & $\begin{array}{l}\text { Shunt resistance to represent the total resistance for the } \\
\text { current leakage in the } \mathrm{P}-\mathrm{N} \text { junction of the solar cell }\end{array}$ \\
\hline $\mathrm{Is}_{1}$ & Current passing through the first diode \\
\hline $\mathrm{Is}_{2}$ & Current passing through the second diode \\
\hline K & constant of $=1.380 \times 10^{-23}(\mathrm{~J} / \mathrm{Ko})$ \\
\hline q & $1.602 \times 10^{-19}(\mathrm{C})$ coulombs. \\
\hline $\mathrm{ABC}$ & Artificial bee colony \\
\hline MPSO & Mutant particle swarm optimization \\
\hline SSA & Salp swarm algorithm \\
\hline ITLBO & Improved teaching-learning-based optimization \\
\hline
\end{tabular}

\section{References}

1. Kaliraj, P.; Devi, T. Artificial Intelligence Theory, Models, and Applications, 2nd ed.; 206 B/W Illustrations; Auerbach Publications: Boca Raton, FL, USA, 2021; p. 506.

2. He, Q.; Zheng, H.; Ma, X.; Wang, L.; Kong, H.; Zhu, Z. Artificial intelligence application in a renewable energy-driven desalination system: A critical review. Energy AI 2022, 7, 100123. [CrossRef]

3. Sarita, K.; Devarapalli, R.; Rai, P. Modeling and control of dynamic battery storage system used in hybrid grid. Energy Storage 2020, 2, e146. [CrossRef]

4. Surendra, H.H.; Seshachalam, D.; Sudhindra, K.R. Design of Standalone Solar Power Plantusing System Advisor Model in Indian Context. Int. J. Recent Technol. Eng. (IJRTE) 2020, 5, 2277-3878.

5. Baschieri, D.; Magni, C.A.; Marchioni, A. Comprehensive Financial Modeling of Solar PV Systems. In Proceedings of the 37th European Photovoltaic Solar Energy Conference and Exhibition, Lisbon, Portugal, 7-11 September 2020.

6. Qais, M.H.; Hasanien, H.M.; Alghuwainem, S. Transient search optimization for electrical parameters estimation of photovoltaic module based on datasheet values. Energy Convers. Manag. 2020, 214, 112904. [CrossRef]

7. Ramadan, A.; Kamel, S.; Korashy, A.; Yu, J. Photovoltaic Cells Parameter Estimation Using an Enhanced Teaching Learning Based Optimization Algorithm. Iran. J. Sci. Technol. 2019, 44, 767-779. [CrossRef]

8. Piliougine, M.; Guejia-Burbano, R.A.; Petrone, G.; Sánchez-Pacheco, F.J.; Mora-López, L.; Sidrach-de-Cardon, M. Parameters extraction of single diode model for degraded photovoltaic modules. Renew. Energy 2021, 164, 674-686. [CrossRef]

9. Ramadan, A.; Kamel, S.; Ibrahim, A.A. Parameters Estimation of Photovoltaic Cells Using Self-adaptive Multi-population Rao Optimization Algorithm. Aswan Univ. J. Sci. Technol. 2021, 31, 34. 
10. Stornelli, V.; Muttillo, M.; de Rubeis, T.; Nardi, I. A New Simplified Five-Parameter Estimation Method for Single-Diode Model of Photovoltaic Panels. Energies 2019, 12, 4271. [CrossRef]

11. Messaoud, R.B. Extraction of uncertain parameters of double-diode model of a photovoltaic panel using Ant Lion Optimization Appl. Sci. 2018, 2, 239. [CrossRef]

12. Ramadan, A.; Kamel, S.; Taha, I.B.M.; Tostado-Véliz, M. Parameter Estimation of Modified Double-Diode and Triple-Diode Photovoltaic Models Based on Wild Horse Optimizer. Electronics 2021, 10, 2308. [CrossRef]

13. Suganya, T.; Rajendran, V.; Mangaiyarkarasi, P. "Parameters Extraction of the Double Diode Model for the Polycrystalline Silicon Solar Cells" Advances in Computing and Data Sciences. ICACDS 2021. Communications in Computer and Information Science; Springer: Berlin/Heidelberg, Germany, 2021; Volume 1440.

14. Yaqoob, S.J.; Saleh, A.L.; Motahhir, S.; Agyekum, E.B.; Nayyar, A.; Qureshi, B. Comparative study with practical validation of photovoltaic monocrystalline module for single and double diode models. Sci. Rep. 2021, 11, 19153. [CrossRef]

15. Ramadan, A.; Kamel, S.; Hussein, M.M.; Hassan, M.H. A New Application of Chaos Game Optimization Algorithm for Parameters Extraction of Three Diode Photovoltaic model. IEEE Access 2021, 9, 51582-51594. [CrossRef]

16. Houssein, E.H.; Zaki, G.N.; Diabb, A.A.Z.; Younis, E.M. An efficient Manta Ray Foraging Optimization algorithm for parameter extraction of three-diode photovoltaic model. Comput. Electr. Eng. 2021, 94, 107304. [CrossRef]

17. Ramadan, A.; Kamel, S.; Khurshaid, T.; Oh, S.R.; Rhee, S.B. Parameter Extraction of Three Diode Solar Photovoltaic Model Using Improved Grey Wolf Optimizer. Sustainability 2021, 13, 6963. [CrossRef]

18. Qin, L.; Xie, S.; Yang, C.; Cao, J. “Dynamic model and dynamic characteristics of solar cell” Conference Paper in Zhongguo Dianji Gongcheng Xuebao. In Proceedings of the Chinese Society of Electrical Engineering, Melbourne, Australia, 3-6 June 2013.

19. Yousri, D.; Allam, D.; Eteibaa, M.B.; Suganthanb, P.N. Static and dynamic photovoltaic models' parameters identification using Chaotic Heterogeneous Comprehensive Learning Particle Swarm Optimizer variants. Energy Convers. Manag. 2019, 182, 546-563. [CrossRef]

20. Maniraj, B.; Fathima, A.P. Parameter extraction of solar photovoltaic modules using various optimization techniques: A review. J. Phys. Conf. Ser. 2020, 1716, 012001. [CrossRef]

21. Venkateswari, R.; Rajasekar, N. Review on parameter estimation techniques of solar photovoltaic systems. Int. Trans. Electr. Energ. Syst. 2021, 31, e13113. [CrossRef]

22. Soliman, M.A.; Al-Durra, A.; Hasanien, H.M. Electrical Parameters Identifica-tion of Three-Diode Photovoltaic Model Based on Equilibrium Optimizer Algorithm. IEEE Access 2021, 9, 41891-41901. [CrossRef]

23. Joshi, H.; Arora, S. Enhanced Grey Wolf Optimization Algorithm for Global Optimization. Fundam. Inform. 2017, 153, 235-264. [CrossRef]

24. Tao, Q.; Guo, H.; Li, J.; Gao, K.; Han, Y. Improved migrating birds optimization algorithm to solve hybrid flowshop scheduling problem with lot-streaming. Inst. Electr. Electron. Eng. Access 2020, 8, 89782-89792.

25. Cheng, J.; Zhao, W. Chaotic enhanced colliding bodies optimization algorithm for structural reliability analysis. Adv. Struct. Eng. 2020, 23, 438-453. [CrossRef]

26. Alghamdi, M.A.; Khan, M.F.N.; Khan, A.K.; Khan, I.; Ahmed, A.; Kiani, A.T.; Khan, M.A. PV Model Parameter Estimation Using Modified FPA With Dynamic Switch Probability and Step Size Function. IEEE Access 2021, 9, 42027-42044.

27. Zhaoa, W.; Wanga, L.; Seyedali, M. Artificial hummingbird algorithm: A new bio-inspired optimizer with its engineering applications. Comput. Methods Appl. Mech. Eng. 2022, 388, 114194. [CrossRef]

28. Naik, M.K.; Panda, R.; Abraham, A. Adaptive opposition slime mould algorithm. Soft Comput. 2021, 25, 14297-14313. [CrossRef]

29. Zhao, W.; Wang, L.; Zhang, Z. Supply-demand-based optimization: A novel economics-inspired algorithm for global optimization. IEEE Access 2019, 7, 73182-73206. [CrossRef]

30. Naruei, I.; Keynia, F. Wild horse optimizer: A new meta-heuristic algorithm for solving engineering optimization problems. Eng. Comput. 2021, 1-32. [CrossRef]

31. Kaur, S.; Awasthi, L.K.; Sangal, A.L.; Dhiman, G. Tunicate Swarm Algorithm: A new bio-inspired based metaheuristic paradigm for global optimization. Eng. Appl. Artif. Intell. 2020, 90, 103541. [CrossRef]

32. Allam, D.; Yousri, D.A.; Eteiba, M.B. Parameters extraction of the three diode model for the multi-crystalline solar cell/module using Moth-Flame Optimization Algorithm. Energy Convers. Manag. 2016, 123, 535-548. [CrossRef]

33. Hafeez, G.; Javaid, N.; Iqbal, S.; Khan, F.A. Optimal Residential Load Scheduling Under Utility and Rooftop Photovoltaic Units. Energies 2018, 11, 611. [CrossRef] 